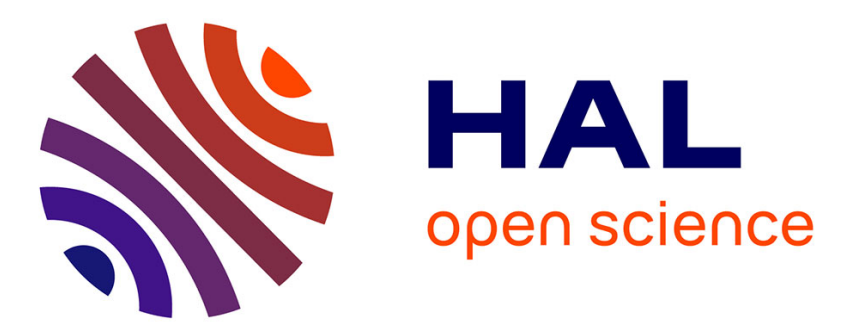

\title{
Self-adaptable hierarchical clustering analysis and differential evolution for optimal integration of renewable distributed generation
}

Rodrigo Mena, Martin Hennebel, Yan-Fu Li, Enrico Zio

\section{To cite this version:}

Rodrigo Mena, Martin Hennebel, Yan-Fu Li, Enrico Zio. Self-adaptable hierarchical clustering analysis and differential evolution for optimal integration of renewable distributed generation. Applied Energy, 2014, 133, pp.388-402. 10.1016/j.apenergy.2014.07.086 . hal-01090342

HAL Id: hal-01090342

https://hal-centralesupelec.archives-ouvertes.fr/hal-01090342

Submitted on 3 Dec 2014

HAL is a multi-disciplinary open access archive for the deposit and dissemination of scientific research documents, whether they are published or not. The documents may come from teaching and research institutions in France or abroad, or from public or private research centers.
L'archive ouverte pluridisciplinaire HAL, est destinée au dépôt et à la diffusion de documents scientifiques de niveau recherche, publiés ou non, émanant des établissements d'enseignement et de recherche français ou étrangers, des laboratoires publics ou privés. 

Differential Evolution for Optimal Integration of Renewable Distributed Generation

\author{
Rodrigo Mena ${ }^{\mathrm{a}}$ \\ rodrigo.mena@ecp.fr
}

Tel. (33) 1141131307

\author{
Martin Hennebel ${ }^{\mathrm{c}}$ \\ martin.hennebel@supelec.fr
}

\author{
Yan-Fu Li $\mathbf{i}^{\mathrm{a}}$ \\ yanfu.li@ecp.fr \\ yanfu.li@supelec.fr
}

\author{
Enrico Zio ${ }^{\text {ab* }}$ \\ enrico.zio@ecp.fr \\ enrico.zio@supelec.fr \\ enrico.zio@polimi.it
}

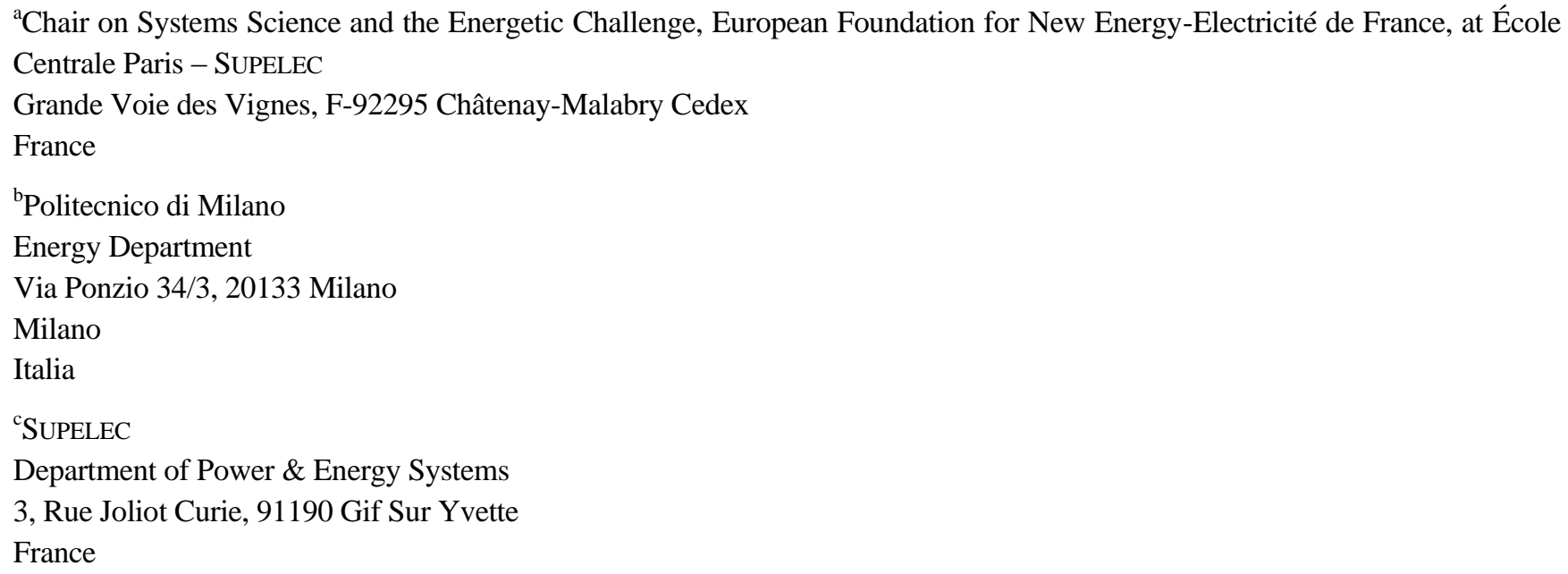

\section{Abstract}

In a previous paper, we have introduced a simulation and optimization framework for the integration of renewable generators into an electrical distribution network. The framework searches for the optimal size and location of the distributed renewable generation units (DG). Uncertainties in renewable resources availability, components failure and repair events, loads and grid power supply are incorporated. A Monte Carlo simulation - optimal power flow (MCS-OPF) computational model is used to generate scenarios of the uncertain variables and evaluate the network electric performance with respect to the expected value of the global cost $(E C G)$. The framework is quite general and complete, but at the expenses of large computational times for the analysis of real systems. In this respect, the work of the present paper addresses the issue and introduces a purposely tailored, original technique for reducing the computational efforts of the analysis. The originality of the proposed approach lies in the development of a new search engine for performing the minimization of the $E C G$, which embeds hierarchical clustering analysis (HCA) within a differential evolution (DE) search scheme to identify groups of similar individuals in the DE population and, then, $E C G$ is calculated for selected representative individuals of the groups only, thus reducing the number of objective function evaluations. For exemplification, the framework is applied to a distribution network derived from the IEEE 13 nodes test feeder. The results show that the newly proposed hierarchical clustering differential evolution (HCDE) MCS-OPF framework is effective in finding optimal DG-integrated network configurations with reduced computational efforts. 
35 distributed renewable generation, uncertainty, simulation, optimization, differential evolution, hierarchical 36 clustering analysis

\section{ACRONYMS}

$\begin{array}{llll}\text { DE } & \text { Differential evolution } & \text { MCS } & \text { Monte Carlo simulation } \\ \text { DG } & \text { Distribution generation } & \text { MS } & \text { Main supply } \\ \text { EA } & \text { Evolutionary algorithm } & \text { OPF } & \text { Optimal power flow } \\ \text { EV } & \text { Electric vehicle } & \text { PSO } & \text { Particle swarm optimization } \\ \text { GA } & \text { Genetic algorithm } & \text { PV } & \text { Photovoltaic } \\ \text { HCA } & \text { Hierarchical clustering analysis } & \text { ST } & \text { Storage device } \\ \text { HCDE } & \text { Hierarchical clustering analysis differential evolution } & \text { W } & \text { Wind turbine }\end{array}$

\section{NOMENCLATURE}

$A_{i, i^{\prime}}^{F D} \quad$ the ampacity of the feeder $\left(i, i^{\prime}\right)(\mathrm{A})$

$B_{i, i^{\prime}} \quad$ susceptance of the feeder $\left(i, i^{\prime}\right)(1 / \Omega)$

$B G T$ available DG integration budget (\$)

CCC cophenetic correlation coefficient

$C C C_{t h} \quad$ cophenetic correlation coefficient thrshold

$C G \quad$ global cost $(\$ / \mathrm{h})$

$\mathrm{Ci} \quad$ total fixed investment and operation cost (\$)

$c i_{j} \quad$ investment cost of the DG technology type $j(\$)$

Co operating costs of power generation and

distribution $(\$ / \mathrm{h})$

Coc $\quad$ crossover coefficient $\in[0,1]$

Cop opportunity cost for kWh not supplied ( $\$ / \mathrm{kWh})$

$\operatorname{Cov}_{j}^{P S}$ variable operating cost of the power source $j$

$\operatorname{Cov}_{i, i^{\prime}}^{F D}$

$D^{s p}$

$\bar{D}^{s p}$

$d_{p, q}^{s p} \quad$ linkage distance between groups $p$ and $q$

$d_{C O} \quad$ cut off linkage distances

$D G$

$d g$

$d_{\min }$

$d_{N C=4}$

$E C G$ variable operating cost of the feeder $\left(i, i^{\prime}\right)$

matrix of linkage distances between groups at step $s p$

average of $D^{s p}$

set of available types of distribution generation technologies

number of types of available distribution

generation technologies

minimum linkage distance

linkage distances to form at least four clusters

expected global cost $(\$ / \mathrm{h})$ $p_{j}^{+} \quad$ hourly probability distribution of EV discharging state per day

$P a_{i, j}^{P S} \quad$ available power in power source of type $\mathrm{j}$ allocated at node

$p_{d \% \text { tile }} \quad$ linkage distances percentile

$P_{i, j}^{M S}$

$P_{c a p_{j}}^{M S}$

$P_{R_{j}}^{E V}$

$P_{R_{j}}^{S T}$

$P_{R_{j}}^{W}$

$P u_{i, j}^{P S}$

POP

$P S$

$P V$

$p s$

$Q_{i, j}^{S T}$

$s_{i}$

$S E_{j}^{S T}$

power supply of MS type $j$ at node $i(\mathrm{~kW})$

maximum capacity of the MS type $j(\mathrm{~kW})$

rated power of EV technology type $j(\mathrm{~kW})$

rated power of ST technology type $j(\mathrm{~kW})$

rated power of $\mathrm{W}$ technology type $j(\mathrm{~kW})$

used power from the power source type $j$ at node $i$

population

set of all types of power sources

set of solar photovoltaic technologies

number of all types of available power generation technologies

level of charge in ST type $j$ at node $i(\mathrm{~kJ})$

solar irradiance at node $i \in[0,1]$

ST $\quad$ set of storage devices technologies

$T_{a_{i}} \quad$ ambient temperature at node $i\left({ }^{\circ} \mathrm{C}\right)$

$t_{d} \quad$ hour of the day (h)

th lifetime of the project (h)

$T L$ total demand of power in the distribution network $(\mathrm{kW})$

$T L_{h} \quad$ highest total demand of power in the distribution network $(\mathrm{kW})$ 


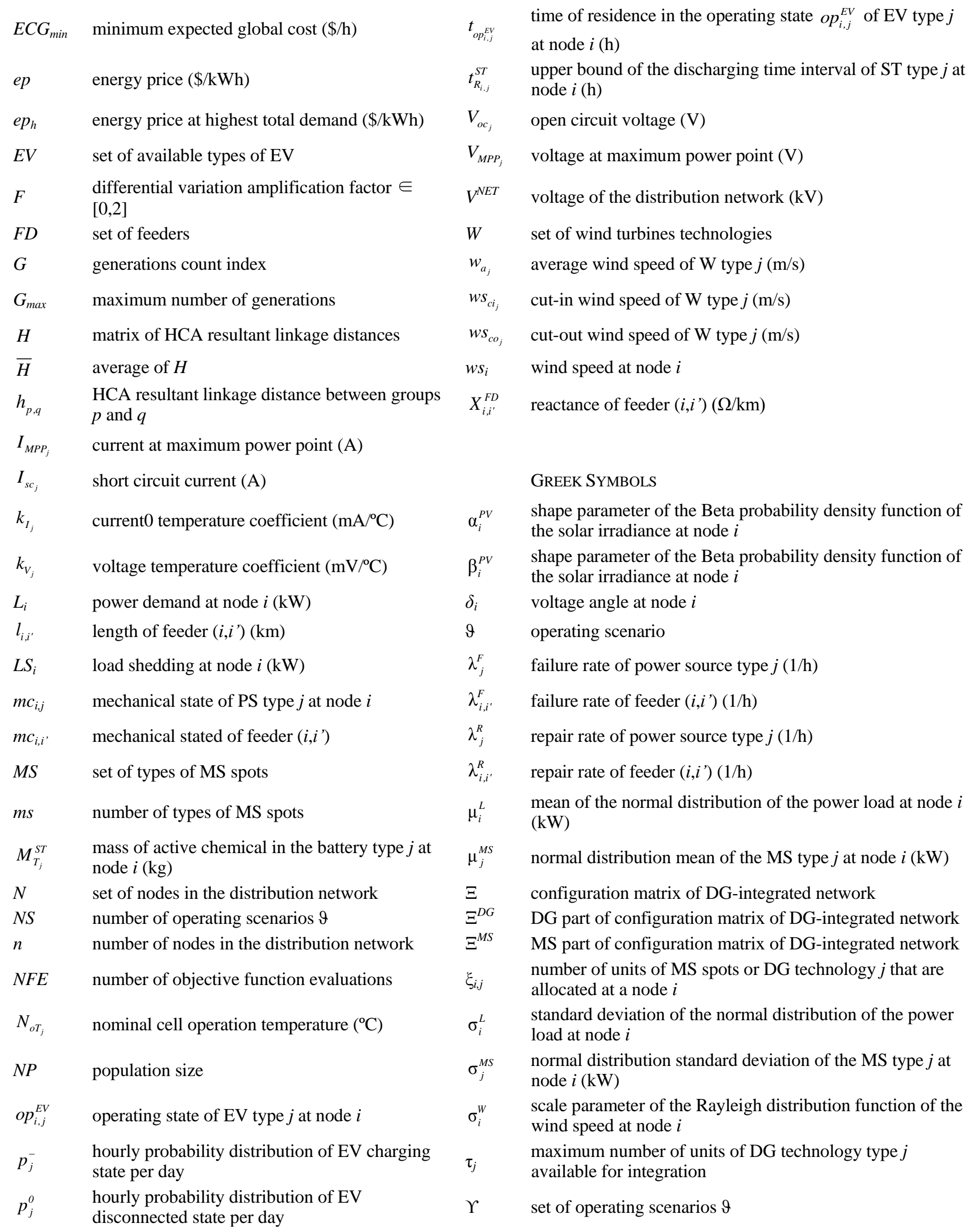


Renewable distribution generation (DG) requires the selection of the different available technologies, and their sizing and allocation onto the power distribution network, considering the specific economic, operational and technical constraints [1-5]. This can become a complex optimization problem, depending on the size of the distribution network and the number of renewable DG technologies available, that can lead to combinatorial explosion [1, 3, 6-9]. Furthermore, for each renewable DG plan considered, the power flow problem needs to be solved to assess the response of the distribution network in terms of power and voltage profiles, available power usage, power demand satisfaction, economic performances, etc., with possibly significant computation times.

Heuristic optimization techniques belonging to the class of Evolutionary Algorithms (EAs), like honey bee mating [10], particle swarm optimization (PSO) [9, 11-13], differential evolution (DE) [14, 15] and genetic algorithms (GA) $[2,3,16,17]$, have been considered for the solution to this problem, since they can deal straightforwardly with non-convex combinatorial problems, discontinuous search spaces and non-differentiable objective functions $[1,9]$.

To improve the performance of EAs for the complex optimization problem of DG planning, we consider the integration of clustering [18-23]. This can be directed to the enhancement of the global and/or local searching ability of the algorithm, and amounts to identifying groups of similar individuals and applying different evolution operators to those of a same cluster (group) [18, 20-22], e.g. for random generation of new individuals in the neighborhood of cluster centroids [23], or multi-parents crossover over new randomly generated individuals spread in the global feasible space [19]. Even if convergence is improved, some of these methodologies increase temporarily the overall size of the population and, thus, the computational effort. In addition, the accuracy of the clusters structures in representing the distribution of individuals must be controlled for performing clustering conveniently.

The main original contribution of the work here presented, lies in the development of the clustering strategy in a controlled manner. The implementation of such clustering strategy is done within a Monte Carlo simulation and optimal power flow (MCS-OPF) model and differential evolution (DE) optimization framework [24] previously developed by the authors for the integration of renewable generators into an electrical distribution network: the framework searches for the optimal size and location of the distributed renewable generation units (DG) [25]. Optimality of the DG plan is sought with respect to the expected global cost $(E C G)$. The introduction of the clustering is hierarchically (i.e., hierarchical clustering analysis, HCA, [26]) by a controlled way of reducing the number of individuals to be evaluated during the DE search, therefore, improving the computational efficiency. Henceforth, we call our method hierarchical clustering differential evolution (HCDE).

HCA is introduced to build a hierarchical structure of grouping individuals of the population that present closeness under the control of a specific linkage criterion based on defined distance metrics [26]. The HCA outcomes are the linkage distances at which the grouping actions take place, defining the different levels in the hierarchical structure. 
Two control parameters are introduced in the HCA, the cophenetic correlation coefficient $(C C C)$ and a percentile of the set of linkage distances in the hierarchical structure of the groups $\left(p_{d \%}\right.$ tile). The $C C C$ is a similarity coefficient that measures how representative is the proposed grouping structure by comparing their linkage distances with the original distances between all the individuals in the population. In the hierarchical structure, the linkage distance given by $p_{d \% \text { tile }}$ sets the level at which the groups formed below it are considered to be 'close enough' to constitute independent clusters. The two parameters allow HCDE to adapt itself in each generation of the search, 'deciding' whether to perform clustering if the $C C C$ is greater than or equal to a preset threshold $\left(C C C_{t h}\right)$ and cutting the hierarchical structure in independent clusters according to the linkage distance given by $p_{d \%}$ tile. Then, the individual closest to the centroid of each cluster is taken as the feasible representative solution in the population that enters the evolution phase of the HCDE algorithm. Figure 1 summarizes schematically the structure of the proposed

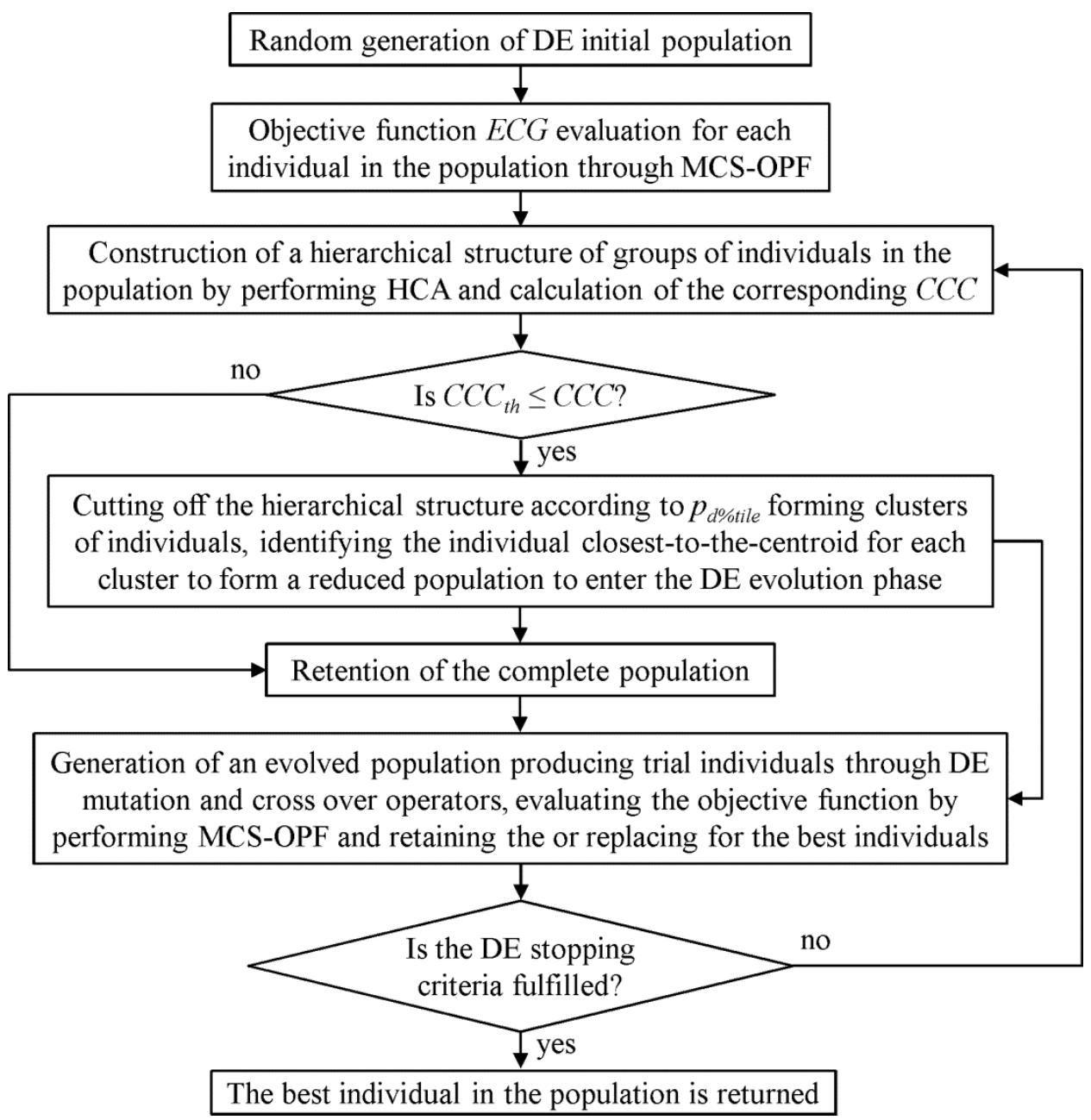

We test the approach on a case study based on the IEEE 13 nodes test feeder distribution network [27], completing the study with a sensitivity analysis to investigate the effects of the parameters controlling the clustering, namely 
For practical ease of the presentation of the approach, in the next section we provide the basic elements of the model of the distribution network considered as case study and we briefly summarize the MCS-OPF model taken from [25]. In Section 3, we embed this in the HCDE for renewable DG selection, sizing and allocation. Finally, in Section 4 we present the numerical results of the case study and in Section 5 we draw some conclusions on the work performed.

\section{RENEWABLE DG-INTEGRATED NETWORK MODEL}

The operation of the renewable DG-integrated network is considered to be dictated by the location and magnitude of the power available in the different sources, the loads and the operating states of the components. Uncertainty is present in the states of operation of the components, due to stochasticity of degradation and failures, and in the behavior of the renewable energy sources. These uncertainties have a direct impact on the power available (from the DG units, main supply spots and/or feeders) to satisfy power demands, which are, in turn, also subject to fluctuations. Furthermore, if the distribution network is considered as a 'price taker' entity, the uncertain behavior of the power demand impacts directly over the energy price $[4,5,28]$. Consequently, an attentive modeling of the uncertainties in renewable DG planning is imperative for well-supported decision-making. Monte Carlo simulation (MCS) has already been used to emulate the stochastic operating conditions and evaluate the performance of power distribution networks [19, 28, 29, 32]. In the present paper, non-sequential MCS is used to randomly sample the modeled uncertain variables for a specific renewable DG plan, without dependence on previous operating conditions, characterizing the network operation in terms of location and magnitudes of power available and loads. Then, the performance of the DG-integrated network is evaluated through the optimal power flow model.

\subsection{Monte Carlo and Optimal Power Flow Simulation}

110 In the proposed framework, the renewable DG technologies considered are of four types: solar photovoltaic (PV), 111 wind turbines (W), electric vehicles (EV) and storage devices (ST); these are represented by the set $D G$ that 112 contains all the $d g$ types of technologies. As for main power supply spots or transformers (MS), the set $M S$ 113 indicates the $m s$ different types of MS considered in the network.

114 The DG-integrated network deployment is represented by the location and capacity size of the power sources, as 115 indicated in matrix form in equation (1) below, where $\xi_{i, j}$ indicates the number of units of main supply spots or DG 116 technology $j$ that are allocated at a node $i$ :

$$
\Xi=\left[\begin{array}{ccccc:ccccc}
\xi_{1, l} & \cdots & \xi_{1, j} & \cdots & \xi_{1, m s} & \xi_{1, m s+1} & \cdots & \xi_{1, m s+j} & \cdots & \xi_{1, m s+d g} \\
\vdots & \ddots & \vdots & \ddots & \vdots & \vdots & \ddots & \vdots & \ddots & \vdots \\
\xi_{i, l} & \cdots & \xi_{i, j} & \cdots & \xi_{i, m s} & \xi_{i, m s+1} & \cdots & \xi_{i, m s+j} & \cdots & \xi_{i, m s+d g} \\
\vdots & \ddots & \vdots & \ddots & \vdots & \vdots & \ddots & \vdots & \ddots & \vdots \\
\xi_{n, l} & \cdots & \xi_{n, j} & \cdots & \xi_{n, m s} & \xi_{n, m s+1} & \cdots & \xi_{n, m s+j} & \cdots & \xi_{n, m s+d g}
\end{array}\right]=\left[\Xi^{M S}: \Xi^{D G}\right] \forall \xi_{i, j} \in \mathbb{Z}^{*}, i \in N, j \in P S
$$


117 where, $N$ and $P S=\{M S \cup D G\}$ are the set of nodes in the network and the set of all power sources, whose 118 cardinalities are $n$ and $p s=m s+d g$, respectively.

119 The set of feeders $F D$ is defined by all the pairs of nodes $\left(i, i^{\prime}\right)$ connected by a distribution line $\forall\left(i, i^{\prime}\right) \in N \times N$.

120 The considered uncertain conditions that determine the operation of the DG-integrated network are accounted for using different stochastic models, as summarized in Table 1. The interested reader can consult [25] for further details.

Table 1. Uncertain conditions models in the DG-integrated network operation

\begin{tabular}{|c|c|c|c|c|}
\hline Variable & Nomenclature & States and Units & Model & Parameters \\
\hline Hour of the day & $t_{d}$ & (h) & Discrete uniform distribution & {$[1,24]$} \\
\hline Mechanical state & $\begin{array}{l}m c_{i, j} \\
m c_{i, i^{\prime}}\end{array}$ & $\begin{array}{l}\text { (0): under repair } \\
(1) \text { : operating }\end{array}$ & Two-state Markov & $\begin{array}{r}\lambda_{j}^{F}, \lambda_{j}^{R} \\
\lambda_{i, i^{\prime}}^{F}, \lambda_{i, i^{\prime}}^{R}\end{array}$ \\
\hline Main power supply & $P_{i, j}^{M S}$ & $(\mathrm{~kW})$ & $\begin{array}{l}\text { Truncated normal distribution } \\
O \leq P_{i, j}^{M S} \leq P_{\text {cap }_{j}}^{M S}\end{array}$ & $\begin{array}{l}\mu_{j}^{M S}, \sigma_{j}^{M S} \\
\quad P_{c a p_{j}}^{M S}\end{array}$ \\
\hline Solar irradiance & $s_{i}$ & {$[0,1]$} & Beta distribution & $\alpha_{i}^{P V}, \beta_{i}^{P V}$ \\
\hline Wind speed & $w s_{i}$ & $(\mathrm{~m} / \mathrm{s})$ & Rayleigh distribution & $\sigma_{i}^{W}$ \\
\hline EV operating state & $o p_{i, j}^{E V}$ & $\begin{array}{l}\text { (-1): charging } \\
(0): \text { disconnected } \\
(1): \text { discharging }\end{array}$ & $\begin{array}{l}\text { 'Block groups' } \\
\text { Hourly probability distribution of } \\
\text { EV operating states per day }\end{array}$ & $t_{d}$ \\
\hline ST level of charge & $Q_{i, j}^{S T}$ & $(\mathrm{~kJ})$ & Uniform distribution & {$\left[0, S E_{j}^{S T} \times M_{T_{i, j}}^{S T}\right]$} \\
\hline $\begin{array}{l}\text { Nodal power } \\
\text { demand }\end{array}$ & $L_{i}$ & $(\mathrm{~kW})$ & $\begin{array}{l}\text { Daily nodal load profiles, hourly } \\
\text { normally distributed load. } \\
\text { Truncated normal distribution } \\
O \leq L_{i} \leq \infty\end{array}$ & $\mu_{i}^{L}\left(t_{d}\right), \sigma_{i}^{L}\left(t_{d}\right)$ \\
\hline
\end{tabular}

where $\forall i, i^{\prime} \in N, j \in P S,\left(i, i^{\prime}\right) \in F D, \quad \lambda_{j}^{F}$ and $\lambda_{j}^{R}(1 / \mathrm{h})$ are the failure and repair rates of the power source $j$, respectively, $\lambda_{i, i^{\prime}}^{F}$ and $\lambda_{i, i^{\prime}}^{R}(1 / \mathrm{h})$ are the failure and repair rates of the feeder $\left(i, i^{\prime}\right)$, respectively, $\mu_{j}^{M S}$ and $\sigma_{j}^{M S}$ are the normal distribution mean and standard deviation associated to the main supply $j$ at node $i, P_{c a p_{j}}^{M S}$ is the maximum capacity of the transformer $j(\mathrm{~kW}), \alpha_{i}^{P V}$ and $\beta_{i}^{P V}$ are the parameters of the Beta probability density function of the solar irradiance at node $i, \sigma_{i}^{W}$ is the scale parameter of the Rayleigh distribution function of the wind speed at node $i, S E_{j}^{S T}(\mathrm{~kJ} / \mathrm{kg})$ is the specific energy of the active chemical in the battery type $j, M_{T_{i, j}}^{S T}(\mathrm{~kg})$ is the mass of active chemical in the battery type $j$ at node $i, \mu_{i}^{L}\left(t_{d}\right)$ and $\sigma_{i}^{L}\left(t_{d}\right)$ are the hourly mean and standard deviation of the normal distribution of the power load at node $i$.

132 Concerning the hour of the day $t_{d}(\mathrm{~h})$, sampled from a discrete uniform distribution $\mathrm{U}(1,24)$, the night interval is 133 defined between 22.00 and 06.00 hours. If the value of $t_{d}$ falls in the night interval, there is no solar irradiation.

134 The resulting realization of one operational scenario of duration $t s(\mathrm{~h})$, for the given DG plan denoted by $\{F D, \Xi\}$, 135 consists in the random sampling of each uncertain variable (Table 1), here indicated by the vector $\vartheta$ below: 


$$
\vartheta=\left[t_{d}, m c_{i, j}, m c_{i, i^{\prime}}, L_{i}, P_{i, j}^{M S}, s_{i}, w s_{i}, o p_{i, j}^{E V}, Q_{i, j}^{S T}\right]
$$

136 To evaluate the performance of the distribution network the OPF model receives as input the location and 137 magnitude of the available power in the power sources and demanded at the loads, which are set by the operating 138 conditions defined by $\{F D, \Xi\}$ and $\vartheta$. The nodal power loads $L_{i}$ are directly sampled, whereas the available power 139 in the power sources (MS and DG) depends on the uncertain variables that represent the behavior of the energy 140 sources, the specific technical characteristics of each type of technology and the mechanical states. The available 141 power in each type of power source considered is modeled by the functions summarized in Table 2, for a given 142 configuration $\{F D, \Xi\}$, operating scenario $\vartheta$ and a generic node $i$.

Table 2. Available power functions of the power sources (PS) $[25,29,30]$

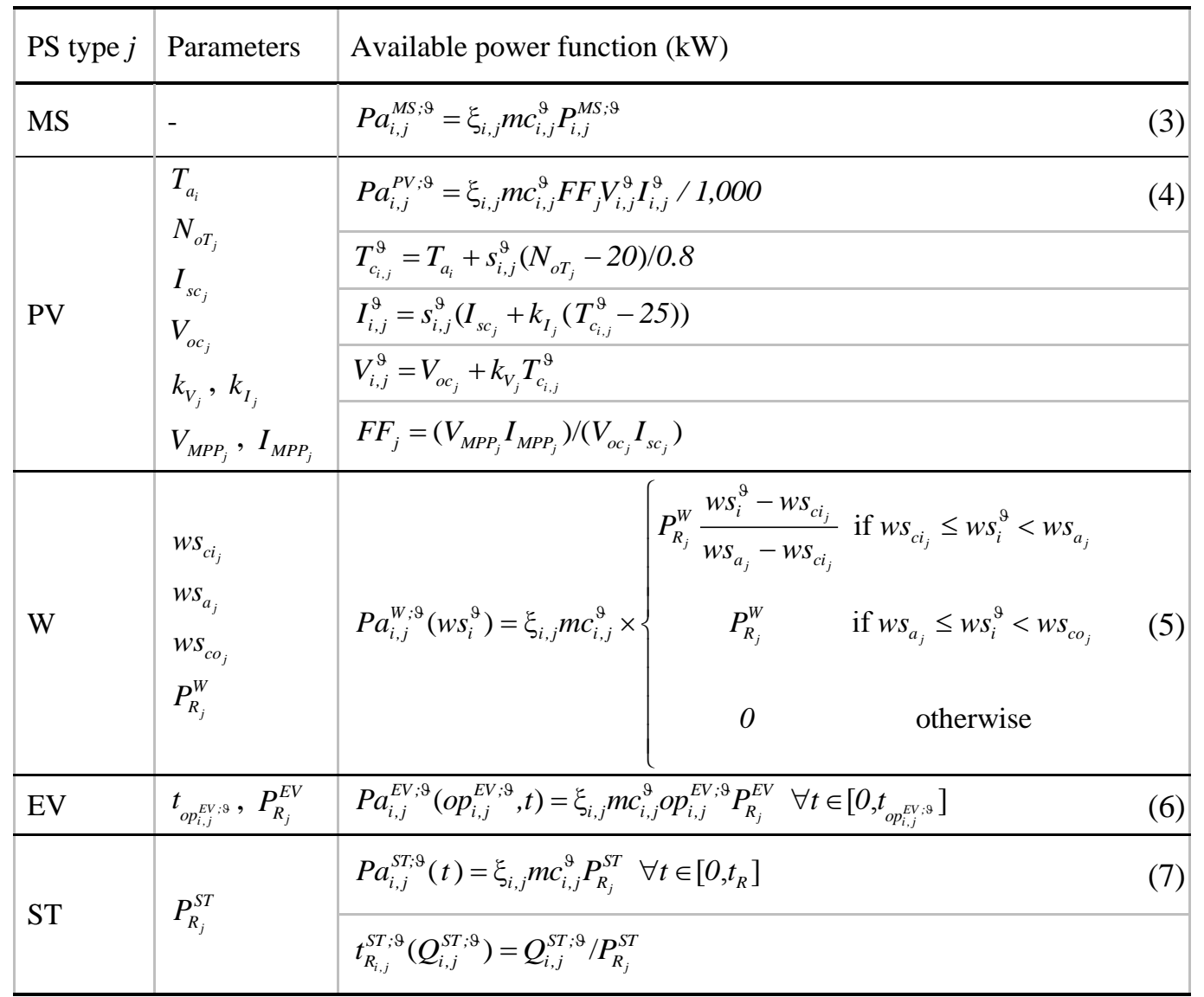

144 In Table 2, $P a_{i, j}^{P S ;}(\mathrm{kW}), \xi_{i, j}$ and $m c_{i, j}^{\vartheta}$ denote the available power, the units and the mechanical state of the power source of type $j$ allocated at node $i$. For solar photovoltaic technologies $j \in P V$, the parameter $T_{a_{i}}\left({ }^{\circ} \mathrm{C}\right)$ is the ambient temperature at node $i, N_{o T_{j}}\left({ }^{\circ} \mathrm{C}\right)$ is the nominal cell operation temperature, $I_{s c_{j}}(\mathrm{~A})$ is the short circuit current, $V_{o c_{j}}(\mathrm{~V})$ is the open circuit voltage, $k_{V_{j}}\left(\mathrm{mV} /{ }^{\circ} \mathrm{C}\right)$ is the voltage temperature coefficient, $k_{I_{j}}\left(\mathrm{~mA} /{ }^{\circ} \mathrm{C}\right)$ is the current temperature coefficients and $V_{M P P_{j}}(\mathrm{~V})$ and $I_{M P P_{j}}(\mathrm{~A})$ are the voltage and current at maximum power point, respectively. For wind turbines of types $j \in W, w s_{c i_{j}}, w s_{a_{j}}$ and $w s_{c o_{j}}(\mathrm{~m} / \mathrm{s})$ are the cut-in, rated and cut-out wind speeds, respectively, and $P_{R_{j}}^{W}(\mathrm{~kW})$ is the rated power of the turbine. For electric vehicles $j \in E V, t_{o p_{i, j}^{E V ;}}(\mathrm{h})$ is the 

is the upper bound of the discharging time interval and $P_{R_{j}}^{S T}(\mathrm{~kW})$ is the rated power. Under the operating conditions set forth, the given configuration of the renewable DG-integrated network $\{F D, \Xi\}$ and the scenario $\vartheta$, the OPF objective is the minimization of the operating cost associated to the generation and distribution of power, considering the revenues per kWh sold. Power flow analysis is performed by DC modeling, neglecting power losses and assuming the voltage throughout the network as constant, linearizing the classic nonlinear power flow formulation by accounting solely for active power flows [31, 32]. The present formulation of the DC optimal power flow problem is:

$$
\min C o^{\vartheta}(P u, \Delta \delta)=\sum_{i \in N} \sum_{j \in P S}\left(\operatorname{Cov}_{j}^{P S}-e p^{\vartheta}\right) P u_{i, j}+\sum_{\left(i, i^{\prime}\right) \in F D} \operatorname{Cov}_{i, i^{\prime}}^{F D}\left|B_{i, i^{\prime}}\left(\delta_{i}-\delta_{i^{\prime}}\right)\right|+\left(\operatorname{Cop}+e p^{\vartheta}\right) \sum_{i \in N} L S_{i}
$$

s.t.

$$
\begin{gathered}
L_{i}^{9}-L S_{i}-\sum_{j \in P S} P u_{i, j}-\sum_{i \in N} m c_{i, i^{\prime}}^{\vartheta} B_{i, i^{\prime}}\left(\delta_{i}-\delta_{i^{\prime}}\right)=0 \\
O \leq P u_{i, j} \leq P a_{i, j}^{P S ; 9} \\
\left|B_{i, i^{\prime}}\left(\delta_{i}-\delta_{i^{\prime}}\right)\right| \leq V^{N E T} A_{i, i^{\prime}}^{F D}
\end{gathered}
$$

160 where $\forall i, i^{\prime} \in N, j \in P S,\left(i, i^{\prime}\right) \in F D$ and the operating scenario $\vartheta, C o^{\vartheta}(\$ / \mathrm{h})$ is the operating cost of the total power supply and distribution, $\operatorname{Cov}_{j}^{P S}(\$ / \mathrm{kWh})$ is the variable operating cost of the power source $j, e p^{9}(\$ / \mathrm{kWh})$ is the energy price, $P u_{i, j}(\mathrm{~kW})$ is the used power from the source of type $j$ at node $i, \operatorname{Cov}_{i, i^{\prime}}^{F D}(\$ / \mathrm{kWh})$ and $B_{i, i^{\prime}}(1 / \Omega)$ are the variable operating cost and the susceptance of the feeder $\left(i, i^{\prime}\right)$, respectively, $\delta_{i}$ is the voltage angle at node $i$, Cop $(\$ / \mathrm{kWh})$ is the opportunity cost for kWh not supplied, $V^{N E T}(\mathrm{kV})$ is the nominal voltage of the network and $A_{i, i^{\prime}}$ (A) is the ampacity of the feeder $\left(i, i^{\prime}\right)$. The load shedding $L S_{i}(\mathrm{~kW})$ is defined as the amount of load disconnected at node $i$ to alleviate congestions in the feeders and/or balance the demand of power with the available power supply.

The distribution network is considered as a 'price taker' entity, assuming a correlation between the total demand of power and the energy price ep $(\$ / \mathrm{kWh})$. Then, the energy price is calculated from an intermediate correlation proposed by $[4,5,28]$ :

$$
e p(T L)=e p_{h}\left(-0.38\left(\frac{T L\left(t_{d}\right)}{T L_{h}}\right)^{2}+1.38 \frac{T L\left(t_{d}\right)}{T L_{h}}\right)
$$

where, $e p_{h}$ is the energy price corresponding to the highest value of total demand considered $T L_{h}$. The total demand of power $T L\left(t_{d}\right)$ at the hour of the day $t_{d}$ is the summation of all the nodal loads $L_{i}\left(t_{d}\right)$ (Table 1$)$.

The constraint given by the equation (9) corresponds to the power balance equation at node $i$, whereas equations (10) and (11) represent the bounds of the power generation and technical limits of the feeders, respectively. 
174 One realization of the MCS-OPF consists of the sampling of NS operating scenarios $\vartheta$ regarded as the set

$175 \Upsilon=\left\{\vartheta_{1}, \ldots, \vartheta_{h}, \ldots, \vartheta_{N S}\right\}$ for each of which the optimal power flow problem is solved, giving in output the values of 176 the minimum operating cost of the total power supply and distribution $C^{\Upsilon}=\left\{C o^{\vartheta_{l}}, \ldots, C o^{\vartheta_{h}}, \ldots, C o^{\vartheta_{N S}}\right\}$.

\section{$177 \quad 2.2 \quad$ Expected Global Cost ECG}

178 The proposed renewable DG-integrated network solutions are evaluated with respect to the expected global cost $179 E C G$. The global cost $C G$ is composed by two terms: the fixed investment and operation (maintenance) costs $C i$ (\$), which are prorated hourly over the life of the project th $(\mathrm{h})$, and the operating costs $C o^{\Upsilon}(\$ / \mathrm{h})$ that is the outcome of the MCS-OPF (equation (8)) described in the precedent Section 2.1. Thus, the global cost function for a scenario $\vartheta$ is given by:

$$
\begin{aligned}
C G^{\vartheta} & =C i+C o^{\vartheta} \forall \vartheta \in \Upsilon \\
C i & =\frac{1}{t h} \sum_{i \in N} \sum_{j \in D G} \xi_{i, j} c i_{j}
\end{aligned}
$$

183

$$
\min E C G^{\Upsilon}
$$

s.t.

$$
\begin{gathered}
\forall \xi_{i, j} \in \mathbb{Z}^{*} \\
\sum_{i \in N} \sum_{j \in D G} \xi_{i, j} c i_{i, j} \leq B G T \\
\sum_{i \in N} \xi_{i, j} \leq \tau_{j} \\
\operatorname{MCS}-\mathrm{OPF}((F D, \Xi), \Upsilon)
\end{gathered}
$$

The meaning of each constraint $\forall i, i^{\prime} \in N, j \in P S,\left(i, i^{\prime}\right) \in F D, \tau_{j} \in \mathbb{Z}^{+}$is: 
- (1): the decision variable $\xi_{i, j}$ is a non-negative integer number.

- (16): the total investment and fixed operation and maintenance costs must be less than or equal to the available budget $B G T$.

- (17): the total number of renewable DG units of each technology $j$ to be allocated must be less than or equal to the maximum number of units available for integration $\tau_{j}$.

- (18): all the equations (8)-(11) of MCS-OPF must be satisfied.

\subsection{Hierarchical Clustering Differential Evolution (HCDE)}

The complex combinatorial optimization problem of DG planning under uncertainties described above is solved by integrating DE with HCA to reduce computational efforts, whereby the evaluation of the objective function is performed by the MCS-OPF presented in Section 3.

$\mathrm{DE}$ is a population-based and parallel, direct search method, shown to be one of the most efficient evolutionary algorithms to solve complex optimization problems [19, 21, 24]. The implementation of the original version of DE involves two main phases: initialization and evolution, summarized below for completeness of the paper [24]:

\section{Initialization}

- Set values of parameters:

- $N P$ : population size

- $G_{\text {max }}$ : maximum number of generations

- Coc: cross over coefficient $\in[0,1]$

- $F$ : differential variation amplification factor $\in[0,2]$

- Generate randomly $N P$ individuals $X$ (decision vectors) within the feasible space, to form the initial population $P O P^{0}=\left\{X_{1}^{0}, \ldots, X_{k}^{0}, \ldots, X_{N P}^{0}\right\}$.

- Evaluate the objective function $f(X)=y$ for each individual

\section{Evolution loop}

- Set generations count index $G=1$

- Set $P O P^{G}=P O P^{O}$

- While $G \leq G_{\max }$ (stopping criterion)

\section{Trial loop}

For each individual $X_{k}^{G}$ in $P O P^{G}, \forall k \in\{1, \ldots, N P\}$ :

- Sample from the uniform distribution three integer indexes $r_{1}, r_{2}, r_{3}$ with $k \neq r_{1} \neq r_{2} \neq r_{3}$ and choose the corresponding three individuals $X_{r_{1}}^{G}, X_{r_{2}}^{G}, X_{r_{3}}^{G}$

- Mutation: generate a mutant individual $V_{k}^{G}$ according to:

$$
V_{k}^{G}=X_{r_{1}}^{G}+F\left(X_{r_{2}}^{G}-X_{r_{3}}^{G}\right)
$$

- Crossover: initialize a randomly generated vector $U_{k}^{G}$, whose dimensionality dim is the same as that of $X_{k}^{G}$ and each coordinate $u_{k ; i}^{G}$ follows a uniform distribution with outcome in $[0,1] \forall i \in\{1, \ldots, d i m\}$. In addition, generate randomly an integer index $r i \in\{1, \ldots, \operatorname{dim}\}$ from a uniform distribution to ensure that at 
least one coordinate from $V_{k}^{G}$ is exchanged to form a trial individual $X T_{k}^{G}$, whose coordinates are defined as follows:

$$
x t_{k, i}^{G}=\left\{\begin{array}{l}
v_{k, i}^{G} \text { if } u_{k, i}^{G} \leq C c o \text { or } i=r i \\
x_{k, i}^{G} \text { if } u_{k, i}^{G}>C c o \text { and } i \neq r i
\end{array}\right.
$$

- Selection: evaluate the objective function for the trial individual $f\left(X T_{k}^{G}\right)$; if $f\left(X T_{k}^{G}\right)<f\left(X_{k}^{G}\right)$ (minimization), then $X T_{k}^{G}$ replaces $X_{k}^{G}$ in the population $P O P^{G}$, otherwise $X_{k}^{G}$ is retained

- Set $G=G+1$

- Once the stopping criterion is reached, sort the individuals in $P O P^{G_{m a x}}$ in descending order according to their values of the objective function and return $X_{1}^{G_{\max }}$.

The original version of DE keeps the population size $N P$ constant, making the computational performance dependent mainly on the number of objective function evaluations carried out during the evolution phase of the algorithm. Then, the integration of HCA into DE is aimed at the reduction of the number of individuals that enter the evolution loop in each generation so as to decrease the number of objective function evaluations.

HCA links individuals or groups of individuals which are similar with respect to a specific property, translated into a metric of distance, obtaining a hierarchical structure. In practice, we use an agglomerative procedure which in $s p$ $=N P-1$ steps fuses the closest pair or individuals or groups of individuals through a linkage function, e.g. single linkage (nearest neighbor distance), complete linkage (furthest neighbor), average linkage, among others, until the complete hierarchical structure is built. The base hierarchical clustering algorithm used in this study can be expressed as follows [26]:

Step 0: Given a population $P O P=\left\{X_{1}, \ldots, X_{k}, \ldots, X_{N P}\right\}$, form the set of singleton groups $O=\left\{O_{p}=\left\{X_{k}\right\}\right\}, \forall p=k \in\{1, \ldots, N P\}$ and calculate the linkage distances between all the $N P$ groups using the average as linkage function and the Euclidean distance as metric:

$$
D^{I}=\left[\begin{array}{ccccc}
d_{1,2}^{l} & \cdots & d_{1, q}^{l} & \cdots & d_{l, N P}^{I} \\
& \ddots & \vdots & \ddots & \vdots \\
& & d_{p, q}^{l} & \cdots & d_{p, N P}^{I} \\
& & & \ddots & \vdots \\
& & & & d_{N P-l, N P}^{I}
\end{array}\right] \forall p, q \in\{1, \ldots, N P\}, k p, k q \in\{1, \ldots, N P\}
$$

where, $d_{p, q}^{l}$ is the average of the Euclidean distances between all the individuals $X_{k}$ belonging to the groups $O_{p}$ and $O_{q}$, respectively.

Step 1: Fuse the first pair of groups $O_{p^{\prime}}$ and $O_{q^{\prime}}$, for which $d_{p^{\prime}, q^{\prime}}^{l}$ is the minimum distance $\min \left(D^{l}\right)$ and form a new group $O_{N P+1}=\left\{O_{p^{\prime}} \cup O_{q^{\prime}}\right\}$.

Update the set of groups $O$ replacing $O_{p}$, and $O_{q}$, by $O_{N P+l}$, and calculate the linkage distances $D^{2}$ between all the $N P-1$ groups in $O$ using (21). 
Step 2: Fuse the second pair of groups $O_{p^{\prime}}$ and $O_{q^{\prime}}$ for which $d_{p^{\prime}, q^{\prime}}^{2}$ is the minimum distance $\min \left(D^{2}\right)$, and form a new group $O_{N P+2}=\left\{O_{p^{\prime}} \cup O_{q^{\prime}}\right\}$.

As in the preceding step, update the set of groups $O$ and calculate the linkage distances $D^{3}$ between all the $N P$-2 groups in $O$ using (21).

Step NP-1: Fuse the last pair of groups with linkage distance $d_{p^{\prime}, q^{\prime}}^{N P-I}$, forming the last group $O_{2 N P-1}=\left\{O_{p^{\prime}} \cup O_{q^{\prime}}\right\}$ that contains all the individuals $X$.

The outcoming hierarchical (or tree) structure can be reported as a sorted table containing the NP-l linkage distances relative to each pairing action of individuals/groups and be graphically illustrated as a dendrogram. Table 3 and Figure 2 present, respectively, the resultant linkage distances and dendrogram obtained from an example set of $N P=8$ two-dimensional individuals $X$ using the above introduced HCA algorithm.
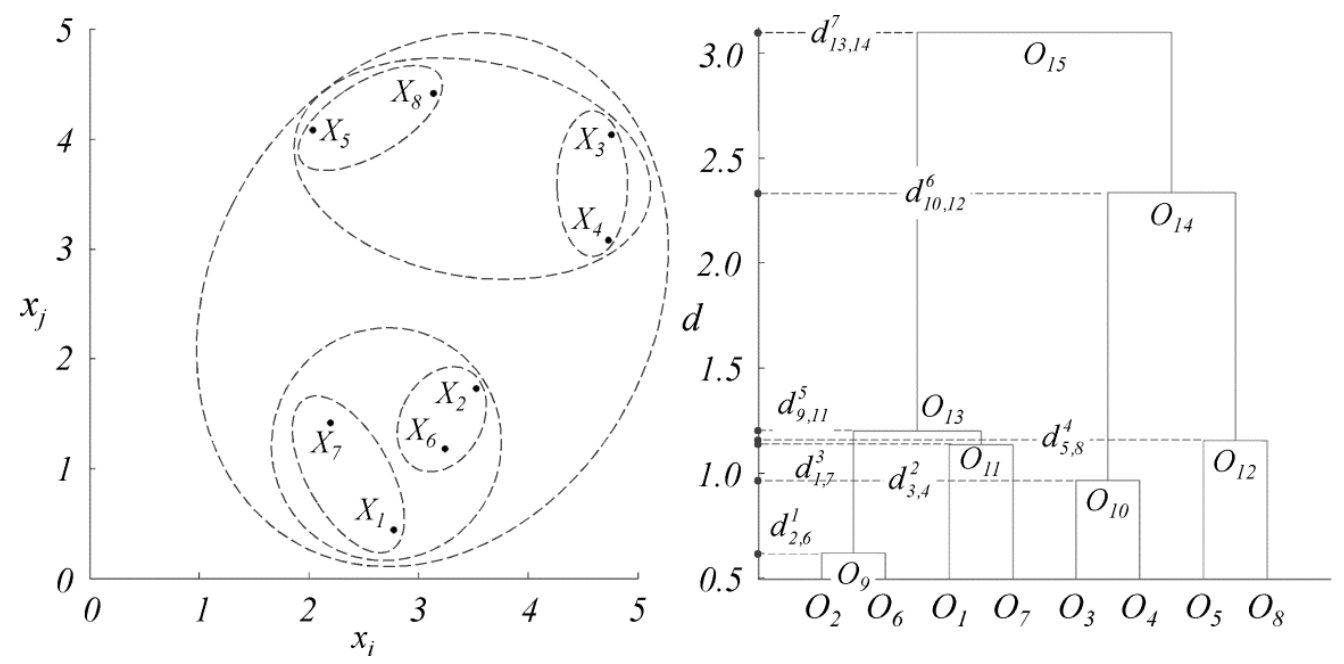

Figure 2. Example dendrogram for average linkage HCA

Table 3. Example hierarchical structure outcome

\begin{tabular}{|c|c|c|c|}
\hline Step $s p$ & Group & Groups linked & Linkage distance $d_{p^{\prime}, q^{\prime}}^{s p}$ \\
\hline 1 & $O_{9}$ & $\left\{O_{2} \cup O_{6}\right\}=\left\{\left\{X_{2}\right\} \cup\left\{X_{6}\right\}\right\}$ & $d_{2,6}^{l}$ \\
\hline 2 & $O_{10}$ & $\left\{O_{3} \cup O_{4}\right\}=\left\{\left\{X_{3}\right\} \cup\left\{X_{4}\right\}\right\}$ & $d_{3,4}^{2}$ \\
\hline 3 & $O_{11}$ & $\left\{O_{1} \cup O_{7}\right\}=\left\{\left\{X_{1}\right\} \cup\left\{X_{7}\right\}\right\}$ & $d_{1,7}^{3}$ \\
\hline 4 & $O_{12}$ & $\left\{O_{5} \cup O_{8}\right\}=\left\{\left\{X_{5}\right\} \cup\left\{X_{8}\right\}\right\}$ & $d_{5,8}^{4}$ \\
\hline 5 & $O_{13}$ & $\left\{O_{9} \cup O_{11}\right\}=\left\{\left\{X_{2}, X_{6}\right\} \cup\left\{X_{1}, X_{7}\right\}\right\}$ & $d_{9,11}^{5}$ \\
\hline 6 & $O_{14}$ & $\left\{O_{10} \cup O_{12}\right\}=\left\{\left\{X_{3}, X_{4}\right\} \cup\left\{X_{5}, X_{8}\right\}\right\}$ & $d_{10,12}^{6}$ \\
\hline 7 & $O_{15}$ & $\left\{O_{13} \cup O_{14}\right\}=\left\{\left\{X_{1}, X_{2}, X_{6}, X_{7}\right\} \cup\left\{X_{3}, X_{4}, X_{5}, X_{8}\right\}\right\}$ & $d_{13,14}^{7}$ \\
\hline
\end{tabular}

As stated above, HCA builds the hierarchical structure through a linkage function introducing in each grouping action a larger or smaller degree of distortion with respect to the original distances between (ungrouped) individuals. The measurement of this distortion is important and the cophenetic correlation coefficient $(C C C)$ is 
introduced to evaluate how representative is the hierarchical structure proposed by the HCA. The $C C C$ can be obtained from equations (22) and (23) below [26].

$$
\begin{aligned}
& C C C=\frac{\sum_{p<q}\left(d_{p, q}^{l}-\bar{D}^{I}\right)\left(h_{p, q}-\bar{H}\right)}{\sqrt{\sum_{p<q}\left(d_{p, q}^{l}-\bar{D}^{I}\right)^{2} \sum_{p<q}\left(h_{p, q}-\bar{H}\right)^{2}}} \forall p, q \in\{1, \ldots, N P\} \\
& H=\left[\begin{array}{ccccc}
h_{1,2} & \cdots & h_{1, q} & \cdots & h_{l, N P} \\
& \ddots & \vdots & \ddots & \vdots \\
& & h_{p, q} & \cdots & h_{p, N P} \\
& & \ddots & \vdots \\
& & & h_{N P-l, N P}
\end{array}\right] \forall p, q \in\{1, \ldots, N P\}, p^{\prime}, q^{\prime} \in\{1, \ldots, 2 N P-1\}, s p \in\{1, \ldots, N P-1\}
\end{aligned}
$$

274 where, $\bar{D}^{1}$ is the mean of the original Euclidean distances $d_{p, q}^{l}$ between all the individuals, $h_{p, q}$ is the linkage 275 distance $d_{p^{\prime}, q^{\prime}}^{s p^{*}}$ where the pair of individuals $X_{p}$ and $X_{q}$ become members of the same group and $\bar{H}$ is the mean of 276 the resultant linkage distances $h_{p, q}$ between all the individuals.

Recalling that the aim of nesting HCA into DE is to increase the computational performance by decreasing the number of individuals to be evaluated in each generation $G$, the presetting of a threshold $C C C_{t h}$ for the $C C C$ value allows defining the level of representativeness required to the hierarchical structure proposed. If the $C C C^{G}$ obtained from applying HCA over the corresponding population $P O P^{G}$ is higher than or equal to the threshold $C C C_{t h}$, the built hierarchical structure is considered an acceptable representation of the original distances amongst the individuals and the selection of a particular partition of the sets of groups can be performed, i.e., the determination of a specific number of clusters. Conversely, if $C C C^{G}$ is less than $C C C_{t h}$, the hierarchical structure is considered not representative enough since it introduces unacceptable distortion that may affect the global searching process in the HCDE.

Whether the hierarchical structure is accepted, the clustering process itself takes place. As before stated, the HCA outcome linkage distances $d_{p^{\prime}, q^{\prime}}^{s p}$ define each level (height) at which a pairing action takes place. If the hierarchical structure is 'cut off' at a specific linkage distance $d_{C O}$, all the groups that are formed below the level $d_{C O}$ become independent clusters. In each generation $G$ of $\mathrm{HCDE}$, a $d_{C O}$ relative to the HCA outcome linkage distances for the corresponding $P O P^{G}$, is determined from a preset percentile $p_{d \%}$ tile of the linkage distances between the minimum $d_{p^{\prime}, q^{\prime}}^{s p}$ that correspond to the first pairing action and the distance to form at least four clusters needed to perform the mutation process in the HCDE. Thus, $d_{C O}$ can be obtained from equations (24) and (25). Figure 3 shows the cutoff distance representation for the example aforementioned, for which the formed clusters are $\left\{O_{2}, O_{6}\right\},\left\{O_{1}\right\},\left\{O_{7}\right\}$, $\left\{O_{3}, O_{4}\right\},\left\{O_{5}\right\}$ and $\left\{O_{8}\right\}$. 


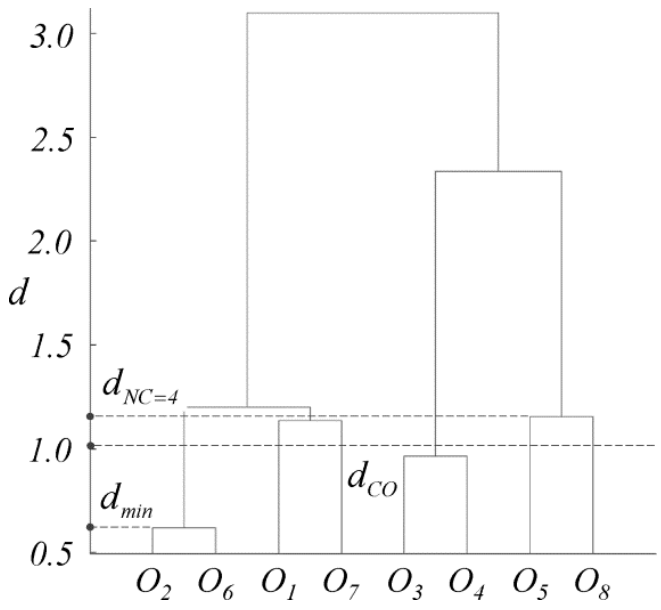

Figure 3. Example of cutoff distance calculation

$$
\begin{gathered}
d_{C O}=d_{\text {min }}+p_{d \% \text { tile }}\left(d_{N C=4}-d_{\text {min }}\right) \\
d_{N C=4}=d_{\left(1-\frac{4}{N P}\right) \% \text { tile }}
\end{gathered}
$$

297 The integration of HCA into DE and the definition of the parameters $C C C_{t h}$ and $p_{d \%}$ tile allow HCDE adaptation at 298 each generation, i.e., deciding whether to perform HCA and determining the clusters to be taken. Then, the 299 individuals closest to the centroids of the formed clusters are considered as the representatives of the group which 300 they belong to and are taken in a reduced population that enters the evolution phase of the HCDE. The proposed 301 HCDE algorithm is summarized schematically in the flowchart of Figure 4. 
Set the values of $N P, G_{\max }, C o c, F, C C C_{t h}$ and $p_{d \%}$ tile

$\downarrow$

Generate randomly $N P$ individuals $\Xi^{D G}$, according to constraints (1), (16)

and (17), to form the initial population $P O P^{0}=\left\{\Xi_{1}^{D G ; 0}, \ldots, \Xi_{k}^{D G ; 0}, \ldots, \Xi_{N P}^{D G ; 0}\right\}$

Evaluate the objective function $E C G^{\mathrm{\gamma}}$ for each individual through $\operatorname{MCS}-\mathrm{OPF}((F D, \Xi), \Upsilon)$ with $\Xi=\left[\Xi^{M S} \mid \Xi^{D G}\right]$

Set generations count index $G=1$

Perform HCA using the average distance linkage function obtaining $d_{p^{\prime}, q^{\prime}}^{s p}$ for the $N P-1$ pairing actions $s p$ and calculate the cophenetic correlation index $C C C^{G}$

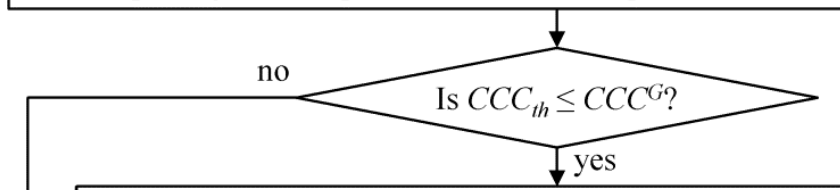

Cut off the hierarchical structure according to $p_{d \% \text { tile }}((24)$ and (25))

forming $N P^{G}$ clusters. Obtain the individual closest-to-the-centroid for each group $c k$ and set $P O P^{\prime G}=\left\{\Xi_{l}^{D G ; G}, \ldots, \Xi_{c k}^{D G ; G}, \ldots, \Xi_{N P^{G}}^{D G ; G}\right\}$

\section{Set $N P^{G}=N P$ and $P O P^{\prime G}=P O P^{G}=\left\{\Xi_{l}^{D G ; G}, \ldots, \Xi_{k}^{D G ; G}, \ldots, \Xi_{N P}^{D G ; G}\right\}$}

Perform the trial loop, from each $\Xi_{k^{\prime}}^{D G ; G} \in P O P^{\prime G}, \forall k^{\prime} \in\left\{1, \ldots, N P^{G}\right\}$ generate a trial individual $\Xi \mathrm{T}_{k^{\prime}}^{G}$ applying mutation and cross over operators ((19) and (20), respectively) and evaluate the objective function $E C G^{\mathrm{\gamma}}\left(\Xi \mathrm{T}_{k^{\prime}}^{G}\right)$

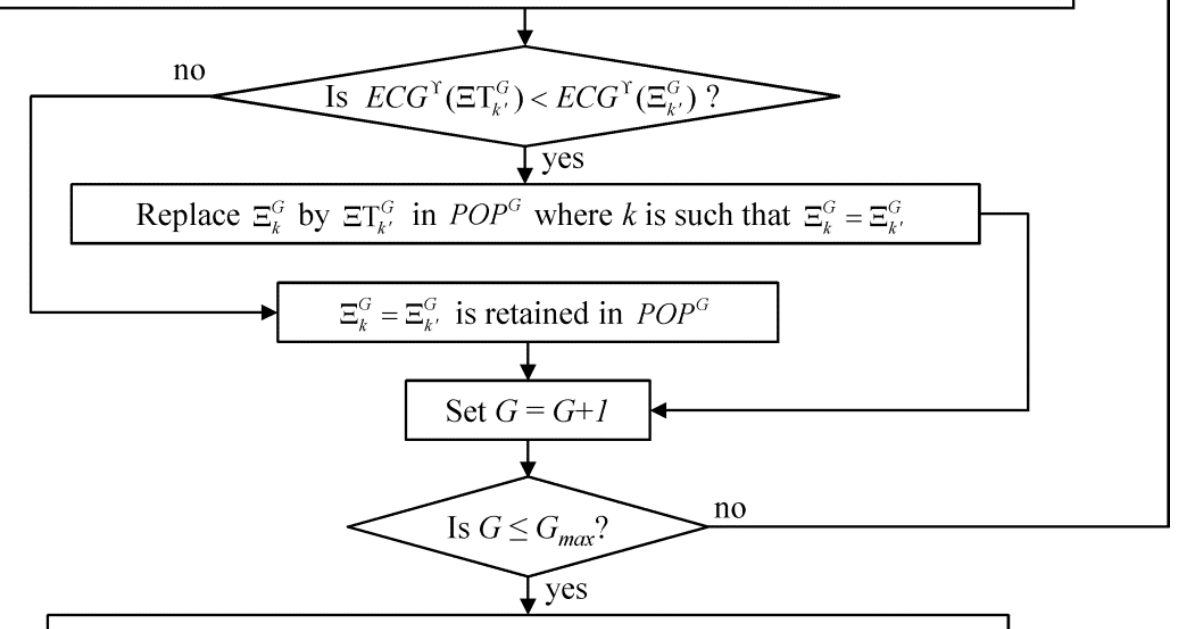

Sort the individuals in $P O P^{G}$ in descending order according to their values of objective function and return $\Xi_{l}^{G_{\max }}$

\section{$304 \quad 4$ CASE STUDY}

305 We consider a modification of the IEEE 13 nodes test feeder distribution network [27] with the original spatial 306 structure but neglecting the feeders of length zero, the regulator, capacitor and switch. The resulting network has 11 307 nodes and presents the relevant characteristics of interest for the analysis, e.g. the presence of a main power supply 308 spot and comparatively low and high spot, and distributed load values [33]. 
310 The distribution network presents a radial structure of $n=11$ nodes as shown in Fig. 1. The nominal voltage $V^{N E T}$ is

$3114.16(\mathrm{kV})$, kept constant for the resolution of the DC optimal power flow problem.

312

313

314

315

316

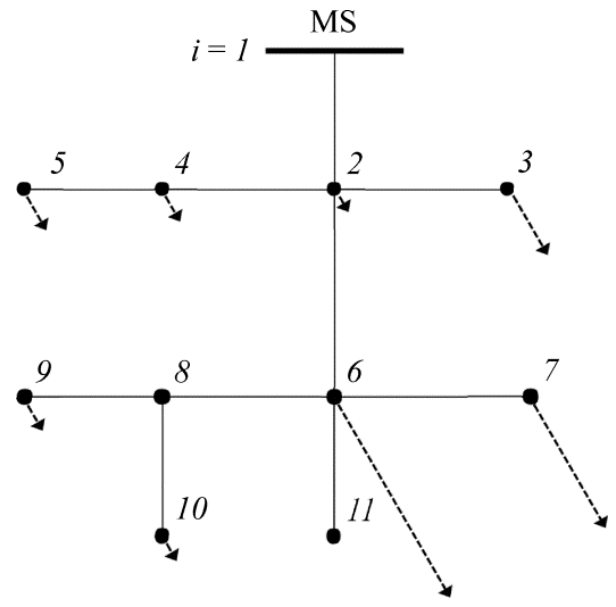

Figure 5. Radial 11-nodes distribution network

Table 4 contains the technical characteristics of the different types of feeders considered: specifically, the indexes of the pairs of nodes $\left(i, i^{\prime}\right)$ that they connect, their length $l$, reactance $X^{F D}$, ampacity $A^{F D}$ and failure and repair rates.

Table 4. Feeders characteristic and technical data [11, 27, 28]

\begin{tabular}{|l|l|l|l|l|l|l|l|c|}
\hline Type & node $i$ & node $i$ & $l(\mathrm{~km})$ & $X^{F D}(\Omega / \mathrm{km})$ & $A^{F D}(\mathrm{~A})$ & $\lambda^{F}(1 / \mathrm{h})$ & $\lambda^{R}(1 / \mathrm{h})$ & Cov $(\$)$ \\
\hline$T 1$ & 1 & 2 & 0.610 & 0.371 & 730 & $3.333 e-04$ & 0.198 & $1.970 e-02$ \\
\hline$T 2$ & 2 & 3 & 0.152 & 0.472 & 340 & $4.050 e-04$ & 0.162 & $9.173 e-03$ \\
\hline$T 3$ & 2 & 4 & 0.152 & 0.555 & 230 & $3.552 e-04$ & 0.185 & $6.205 e-03$ \\
\hline$T 1$ & 2 & 6 & 0.610 & 0.371 & 730 & $3.333 e-04$ & 0.198 & $6.205 e-03$ \\
\hline$T 3$ & 4 & 5 & 0.091 & 0.555 & 230 & $3.552 e-04$ & 0.185 & $6.205 e-03$ \\
\hline$T 6$ & 6 & 7 & 0.152 & 0.252 & 329 & $4.048 e-04$ & 0.164 & $8.904 e-03$ \\
\hline$T 4$ & 6 & 8 & 0.091 & 0.555 & 230 & $3.552 e-04$ & 0.185 & $1.970 e-02$ \\
\hline$T 1$ & 6 & 11 & 0.305 & 0.371 & 730 & $3.333 e-04$ & 0.198 & $1.970 e-02$ \\
\hline$T 5$ & 8 & 9 & 0.091 & 0.555 & 230 & $3.552 e-04$ & 0.185 & $9.173 e-03$ \\
\hline$T 7$ & 8 & 10 & 0.244 & 0.318 & 175 & $3.552 e-04$ & 0.185 & $6.205 e-03$ \\
\hline
\end{tabular}

317 The nodal power demands are built from the load data given in [27] and reported in Figure 6 as daily profiles,

318 normally distributed on each hour $t_{d}$ with mean $\mu^{L}$ and standard deviation $\sigma^{L}$ [29, 34]. 

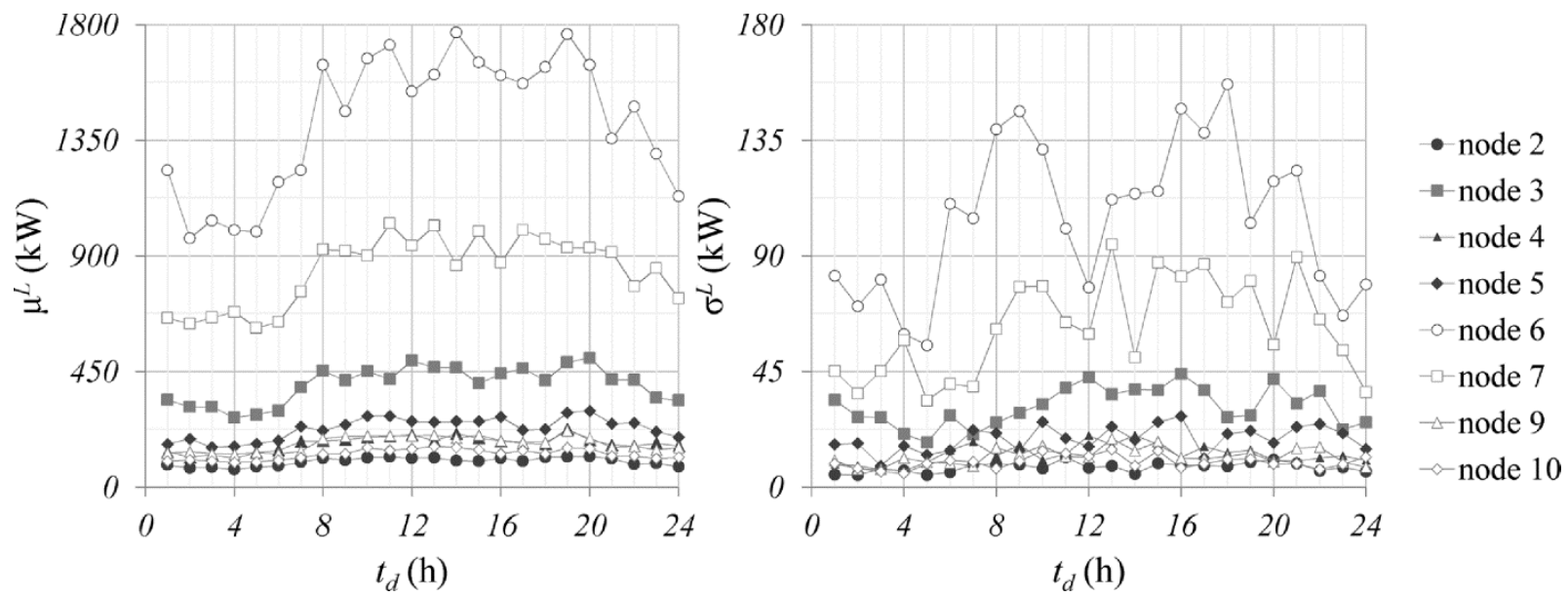

Figure 6. Mean and standard deviation values of normally distributed nodal power demand daily profiles

321 The technical parameters, failure and repair rates and costs of the MS and the four different types of DG technologies (PV, W, EV and ST) available to be integrated into the distribution network are given in Table 5. For the present case study, the distribution region is such that the solar irradiation and wind speed conditions are assumed uniform in the whole network, i.e., the values of the parameters of the corresponding Beta and Rayleigh distributions are assumed constant in the whole network.

Table 5. Power sources parameters and technical data [13, 17, 28, 29, 35-37]

\begin{tabular}{|c|c|c|c|}
\hline Type & Technical parameters & $\begin{array}{l}\text { Distributions parameters, } \\
\text { failure and repair rates }\end{array}$ & Costs \\
\hline$M S$ & $P_{c a p}^{M S}=4250(\mathrm{~kW})$ & $\begin{array}{l}\mu^{M S}=4000(\mathrm{~kW}) \\
\sigma^{M S}=125(\mathrm{~kW}) \\
\lambda^{F}=4.00 e-04(1 / \mathrm{h}) \\
\lambda^{R}=1.30 e-02(1 / \mathrm{h})\end{array}$ & $\operatorname{Cov}=0.145(\$ / \mathrm{kWh})$ \\
\hline$P V$ & $\begin{array}{l}T_{a}=30.00(\mathrm{C}) \\
N_{o T}=43.00(\mathrm{C}) \\
I_{s c}=1.80(\mathrm{~A}) \\
k_{i}=1.40(\mathrm{~mA} / \mathrm{C}) \\
V_{o c}=55.50(\mathrm{~V}) \\
k_{v}=194.00(\mathrm{mV} / \mathrm{C}) \\
V_{M P P}=38.00(\mathrm{~V}) \\
I_{M P P}=1.32(\mathrm{~A})\end{array}$ & $\begin{array}{l}\alpha^{P V}=0.26 \\
\beta^{P V}=0.73 \\
\lambda^{F}=5.00 e-04(1 / \mathrm{h}) \\
\lambda^{R}=1.30 e-02(1 / \mathrm{h})\end{array}$ & $\begin{array}{l}\mathrm{Ci}=48(\$) \\
\mathrm{Cov}=3.76 e-05(\$ / \mathrm{kWh})\end{array}$ \\
\hline$W$ & $\begin{array}{l}w s_{c i}=3.80(\mathrm{~m} / \mathrm{s}) \\
w s_{a}=9.50(\mathrm{~m} / \mathrm{s}) \\
w s_{c o}=23.80(\mathrm{~m} / \mathrm{s}) \\
P_{R}^{W}=50.00(\mathrm{~kW})\end{array}$ & $\begin{array}{l}\sigma^{W}=7.96 \\
\lambda^{F}=6.0 e-04(1 / \mathrm{h}) \\
\lambda^{R}=1.3 e-02(1 / \mathrm{h})\end{array}$ & $\begin{array}{l}C i=113,750(\$) \\
C o v=0.039(\$ / \mathrm{kWh})\end{array}$ \\
\hline$E V$ & $P_{R}^{E V}=6.30(\mathrm{~kW})$ & $\begin{array}{l}\lambda^{F}=2.0 e-04(1 / \mathrm{h}) \\
\lambda^{R}=9.7 e-02(1 / \mathrm{h})\end{array}$ & $\begin{array}{l}C i=17,000(\$) \\
C o v=0.022(\$ / \mathrm{kWh})\end{array}$ \\
\hline$S T$ & $\begin{array}{l}P_{R}^{S T}=0.28(\mathrm{~kW} / \mathrm{kg}) \\
S E=0.04(\mathrm{~kJ} / \mathrm{kg})\end{array}$ & $\begin{aligned} \lambda^{F} & =3.0 e-04(1 / \mathrm{h}) \\
\lambda^{R} & =7.3 e-02(1 / \mathrm{h})\end{aligned}$ & $\begin{array}{l}C i=135.15(\$) \\
C o v=4.62 e-05(\$ / \mathrm{kWh})\end{array}$ \\
\hline
\end{tabular}

327 The hourly per day operating state probability profiles of the EV are presented in Figure 7: $p^{0}, p^{-}$and $p^{+}$correspond to the profiles of disconnected, charging and discharging states, respectively. 


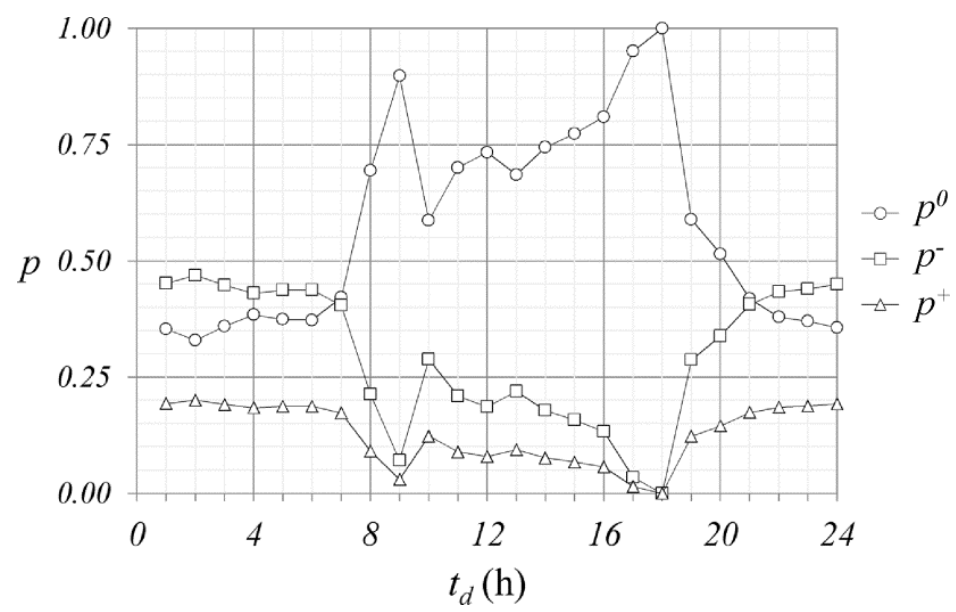

Figure 7. Hourly per day probability data of EV operating states

331 Coherently with constraints (16) and (17), the budget is set to $B G T=4,500,000$ (\$) and the limit of units of the different DG technologies available to be purchased is $\tau=[20000,8,250,10000]$. The maximum value of the energy price is $e p_{h}=0.12(\$ / \mathrm{kWh})[5]$ and the highest value of total demand $T L_{h}$ is set to $4,800(\mathrm{~kW})$. The opportunity cost for kWh not supplied Cop is considered as twice of the maximum energy price.

A total of $N S=500$ random scenarios are simulated by the MCS-OPF with time step $t s=1(\mathrm{~h})$, over a horizon of analysis of 10 years $(t h=87,600(\mathrm{~h}))$, in which the investment and fixed costs are prorated hourly.

The DE iterations are set to perform $G_{\max }=500$ generations over five different cases of population $N P \in\{10,20$, $30,40,50\}$. The differential variation amplification factor $F$ is 1 to maintain the integer-valued definition of the individuals after the mutation, whereas the crossover coefficient Coc is 0.1 .

HCDE runs are performed under the same conditions set for DE $\left(G_{\max }, F\right.$ and $\left.C o c\right)$, but for the population size $N P$ of 50 individuals. A sensitivity analysis is performed over the HCA control parameters, namely the cophenetic correlation coefficient $C C C_{t h}$ and linkage distance percentile $p_{d \% t i l e}$, for all the nine possible pairs $\left(C C C_{t h}, p_{d \% t i l e}\right)$ with $C C C_{\text {th }} \in\{0.6,0.7,0.8\}$ and $p_{d \% \text { tile }} \in\left\{25 \%_{\text {tile }}, 50 \%_{\text {tile, }}, 75 \%_{\text {tile }}\right\}$. Finally, for each of the five DE and nine HCDE settings, twenty realizations are carried out.

\subsection{Results and Discussion}

The results of the DE MCS-OPF for the different population sizes $N P \in\{10,20,30,40,50\}$ are shown in Figure 8. The 50\%tile (median) values of the minimum global costs $E G C_{m i n}$, obtained from each experiment with fixed values of $N P$, are presented as functions of the respective numbers of objective function evaluations $N F E$; the error bars represent the 15 and $85 \%$ tiles.

As expected, for the same number of generations set in the DE MCS-OPF, the larger the population size considered the lower the values of $E G C_{\min }$ obtained (better 'quality' of the minimum). Additionally, we can observe marked tendencies in the reduction of both median and $15-85 \%$ tiles values of $E G C_{\min }$ for increasing $N F E$. Performing a curve fitting over these values, we get: $E G C_{\text {min } ; 50 \% \text { tile }}=49.07 N F E^{-0.13}, E G C_{\text {min } ; 15 \% \text { tile }}=49.07 N F E^{-0.115}$ and 
$E G C_{\text {min } ; 85 \% \text { tile }}=49.07 \mathrm{NFE}^{-.0118}$, with the respective coefficients of determination $R_{50 \% \text { tile }}^{2}=0.994, R_{15 \% \text { tile }}^{2}=0.998$ and $R_{85 \% \text { tile }}^{2}=0.998$. The fact that the difference between the values of the $15-85 \%$ tiles is constant indicates that the dispersion in the $E G C_{\min }(N F E)$ does not depend on $N P$ and can suggest that the global searching performed by the DE is performed homogenously in the feasible space that contains multiple local minima.

358

359

360

361

362

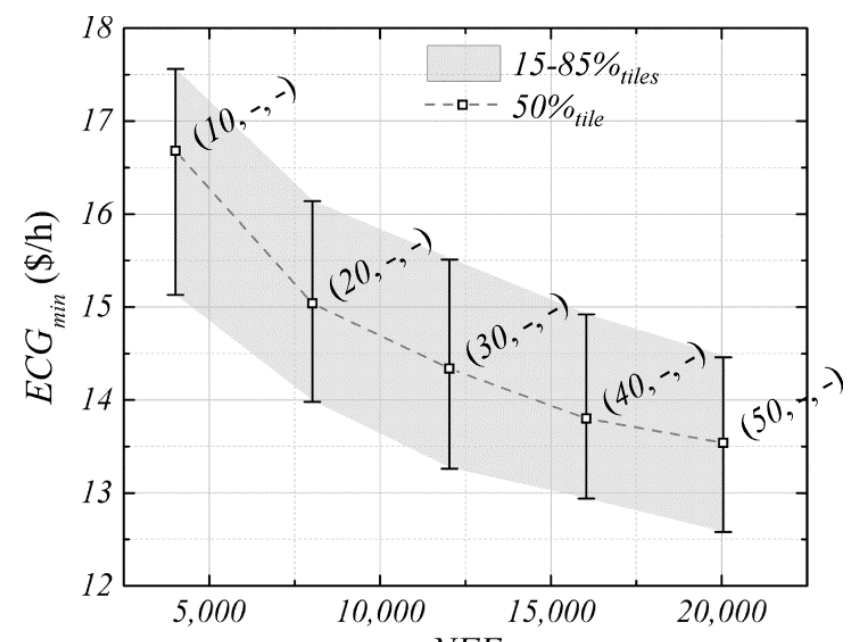

NFE

Figure 8. $E C G_{\min }$ vs $N F E$ for $N P \in\{10,20,30,40,50\}$ set in DE

Figure 9 reports the median $E C G_{\min }$ values corresponding to the HCDE MCS-OPF realizations superposed to the distribution of the median $E C G_{\min }$ and $15-85 \%$ tiles values of the base DE experiments represented by the square markers and shaded area, respectively. The vertical and horizontal error bars account for the 15-85\%tiles of the outcome $E C G_{\min }$ and $N F E$ values.

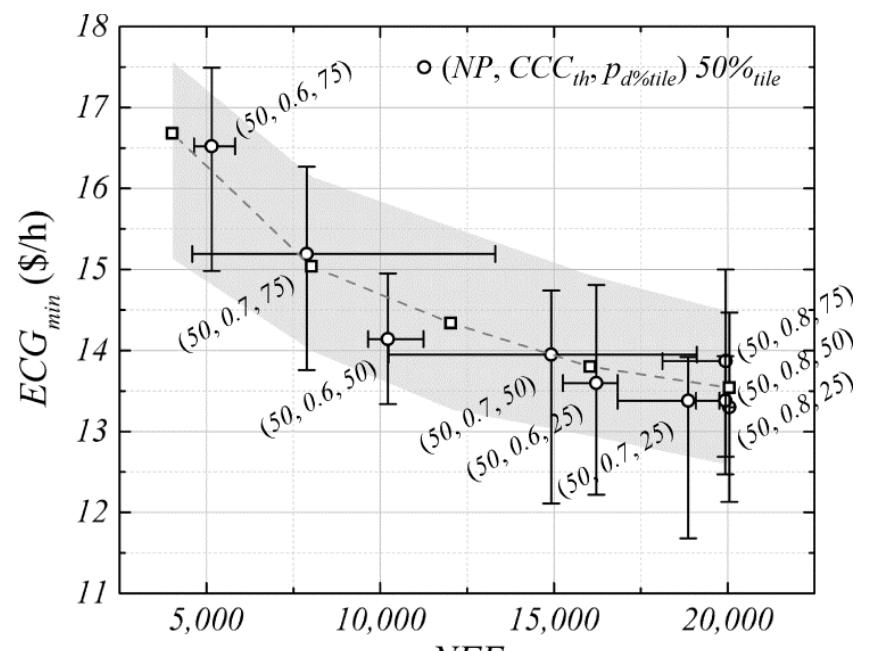

Figure 9. $E C G_{\min }$ vs $N F E$ for each $\left(N P, C C C_{t h}, p_{d \%}\right.$ tile $)$ set in HCDE

Focusing on $C C C_{t h}$, it can be noticed that for the two extreme cases, $C C C_{t h}=0.6$ and 0.8 , the dispersion of the number of objective function evaluations is relatively small. On the contrary, the cases with a $C C C_{t h}=0.7$ present 
Figure 10 shows the median, 15 and $85 \%$ tiles $\mathrm{CCC}$ values as a function of generation $G$ derived from all $\mathrm{HCDE}$ MCS-OPF realizations. On the one hand, recalling that $C C C_{t h}$ is used to control whether it is convenient to perform HCA, the small NFE dispersion in the case with $C C C_{t h}=0.6$ is because clustering is practically been applied in all generations $\left(C C C_{t h} \leq C C C^{G}\right)$, thus disabling any effect generated by passing from populations with original size $N P$ to reduced populations with $N P^{G} \leq N P$ and vice versa. On the other hand, the effect is also being avoided in the case $C C C_{t h}=0.8$ by not applying clustering. Indeed, in Figure 10 it can be observed that after the generation 50 it is unlikely that by performing HCA the proposed hierarchical grouping structures represent well enough the population.

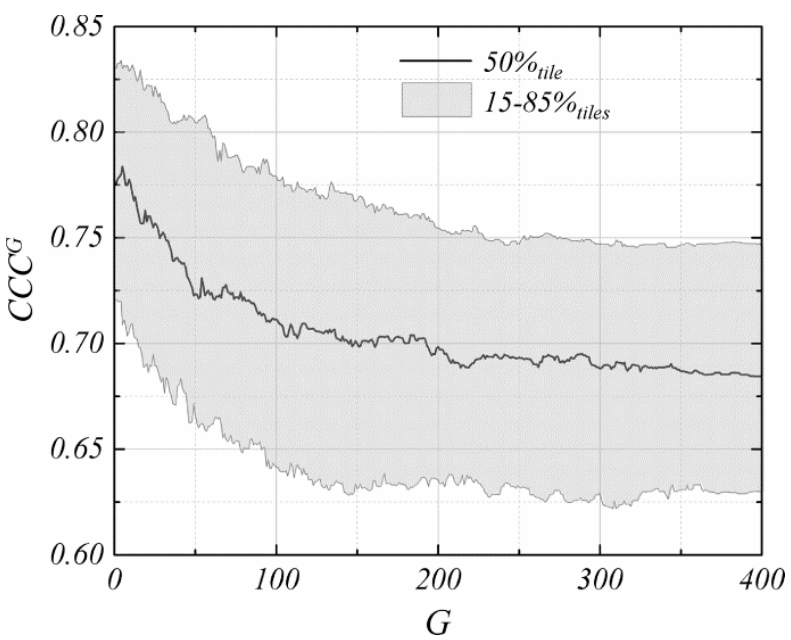

Figure 10. $C C C$ behavior per generation $G$

Differently, the cases for which $C C C_{t h}=0.7$ present high dispersion in the $N F E$ since the median values of $C C C^{G}$ move in the neighborhood of the threshold throughout the major part of the evolution loop in the HCDE. Moreover, in general terms, the values of $C C C^{G} 15-85 \%$ tiles maintain certain symmetry with respect to the median, i.e., performing or not HCA are equally likely events, producing high fluctuations in the number of individuals considered as population and, therefore, affecting in the same way the NFE.

The above mentioned insights are noticeable also in Figure 11, which shows the empirical probability density functions $(p d f \mathrm{~s})$ of the population size $N P^{G}$ per generation for each $\left(N P, C C C_{t h}, p_{d \% t i l e}\right)$ set in HCDE. Indeed, the average probabilities of performing HCA throughout the evolution cycle for the different values of $C C C_{t h}=0.6,0.7$ and 0.8 are $0.98,0.54$ and 0.078 , respectively.

Regarding the percentile of the linkage distance $p_{d \% \text { tile, }}$, in Figure 11 it is possible to identify the three peaks of reduction in the population size, confirming the role of this control parameter in defining the scale at which the hierarchical structures proposed are 'cut off' when the HCA takes place. In fact, lower values of $p_{d \%}$ itile imply smaller reduction in the population size because of the higher demand of proximity between individuals or groups of individuals. In the opposite side, higher values of $p_{d \% t i l e}$ allow forming clusters from individuals or groups which are relatively less similar. 


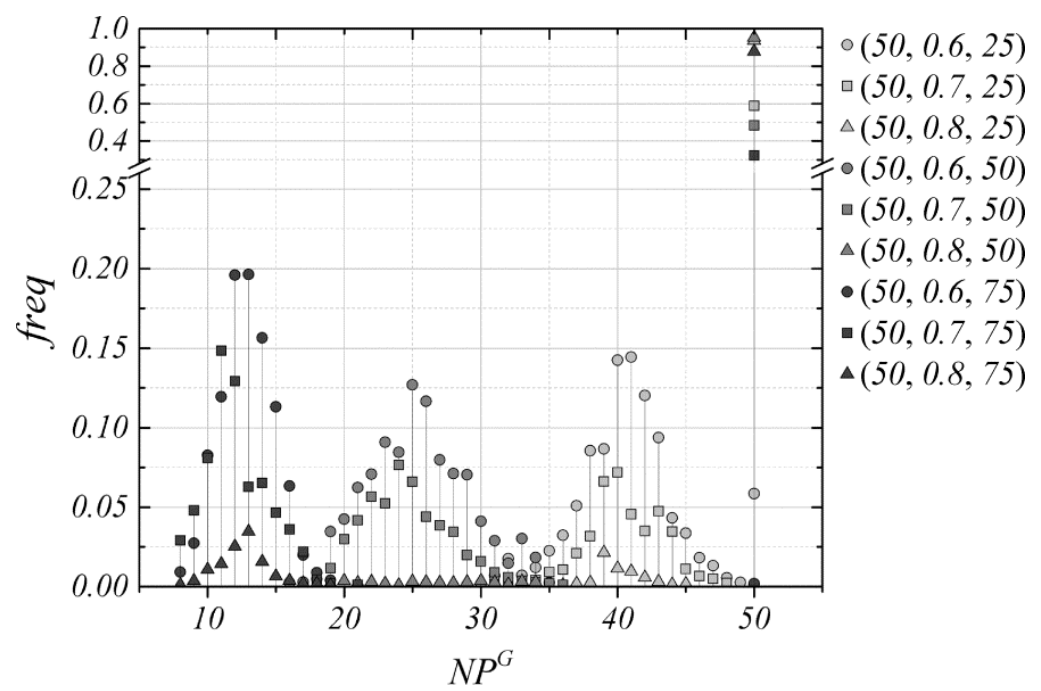

394

395

396

397

398

399

400

401

402

403

404

405

406

407

408

409

410

411

412

413

414

415

416

Figure 11. Empirical $N P^{G} p d f$ for each $\left(N P, C C C_{t h}, p_{d \% t i l e}\right)$ set in HCDE

From the results obtained for all the different DE and HCDE settings, we look for six representative cases for the analysis (Figure 9). From the DE runs, we select the settings with extreme and middle population size $N P \in\{10$, 30, 50\}, whereas from HCDE we choose the cases $\left(N P, C C C_{t h}, p_{d \% t i l e}\right)$ set as $(50,0.6,25),(50,0.6,50),(50,0.7$, $50)$ and $(50,0.7,75)$. The former $(50,0.6,25)$ and $(50,0.6,50)$ cases present significant reductions in the number of $N F E$, with small dispersion and loss of quality of the minimum ECG obtained, compared to the results obtained by diminishing directly the fixed $N P$ in DE from 50 to 10 . Similarly, the cases $(50,0.7,50)$ and $(50,0.7,75)$ may lead to considerable reductions in $N F E$, with acceptable losses of $E C G_{\min }$, but subject to a high degree of variability that compromises the performance.

As for computational times, running on an Intel ${ }^{\circledR}$ Core ${ }^{\mathrm{TM}}$ i7-3740QM (PC) $2.70 \mathrm{GHz}$ without performing parallel computing, the average time to evaluate the objective function is 4.592 (s) for the $N S=500$ scenarios in the MCSOPF; for a fixed population of $N P=50$ and its corresponding $N F E=20,050$, the total time for a single run is on average $25.574(\mathrm{~h})$. Taking into account this, under commonly used hardware configurations, the reductions in computational time that can be achieved by using $\operatorname{HCDE}$ with $(50,0.6,25)$ and $(50,0.6,50)$ settings are $19 \%$ and $49 \%$ for the median, $23 \%$ and $51 \%$ for the $15 \%$ tile, and $16 \%$ and $43 \%$ for the $85 \%$ tile, respectively.

The integration of HCA into the DE algorithm introduces a significant time complexity, conditioning the reductions of computational efforts that can be obtained by applying the proposed HCDE MCS-OPF framework. Indeed, if performing HCA along all generations of DE and running the MCS-OPF on an eventually reduced population (depending on $C C C_{t h}$ and $p_{d \%}$ tile) is computationally heavier than running the MCS-OPF over the complete population, the effects of the framework can be negligible or even negative.

It is possible to formulate the condition to obtain reductions in the computational efforts by the proposed HCDE MCS-OPF framework, from the asymptotic time complexities of the main algorithms that compose it. Table 6 
reports the independent asymptotic time complexities as functions of the generic size $\mathrm{m}$ of the input to each algorithm and of the parameters that define the dimensionality of the HCDE MCS-OPF framework [26, 38].

Table 6. Asymptotic time complexity of the algorithms

\begin{tabular}{|c|c|c|c|c|}
\cline { 2 - 5 } \multicolumn{1}{c|}{} & \multicolumn{4}{c|}{ Algorithm } \\
\cline { 2 - 5 } \multicolumn{1}{c|}{} & PDIST & HC & MCS & OPF \\
\hline \multirow{2}{*}{ Time complexity $T$} & $O\left(d m^{2}\right)^{*}$ & $O\left(m^{2} \log (m)\right)$ & $O(m)$ & $O(\operatorname{size}(A))$ \\
\cline { 2 - 5 } & $O\left(n p s \times N P^{2}\right)$ & $O\left(N P^{2} \log (N P)\right)$ & $O(N S \times n p s)$ & $O\left(N S \times n p s^{2}\right)$ \\
\hline
\end{tabular}

** The matrix A comes from the canonical form $\mathrm{Ax} \leq \mathrm{b}$ of the linear programming of the DC OPF problem approximation

420

421

422

where, $n p s$ represents the size of the DG-integrated network, i.e., the number of nodes $n$ times the number of all the technologies of power generation available $p s, N P$ is the size of the complete population and $N S$ is the number of scenarios in the MCS-OPF.

Comparing the asymptotic time complexities of the algorithms involved in the realization of the proposed framework with and without integrating HCA, the following inequalities must be fulfilled in order to obtain a reduction in the computational time by $\mathrm{HCDE}$ :

$$
\begin{array}{r}
T^{\mathrm{PDIST}}(n p s, N P)+T^{\mathrm{HC}}(N P)+\mathrm{E}\left[N P^{G}\right] \times T^{\mathrm{MCS}-\mathrm{OPF}}(N S, n p s)<N P \times T^{\mathrm{MCS}-\mathrm{OPF}}(N S, n p s) \\
\Downarrow \\
n p s \times N P^{2}+N P^{2} \log (N P)+\mathrm{E}\left[N P^{G}\right] \times N S \times n p s^{2}<N P \times N S \times n p s^{2} \\
\Downarrow \\
\kappa=\frac{N P}{N S \times n p s}+\frac{N P \log (N P)}{N S \times n p s^{2}}+\varepsilon<1 \quad \forall n, p s, N P, N S \in \mathbb{Z}^{*}, \varepsilon=\frac{\mathrm{E}\left[N P^{G}\right]}{N P} \in(0,1]
\end{array}
$$

where, $\varepsilon$ is the expected ratio of the population $N P^{G}$ evaluated along all generations $G$ of DE to the total population $N P$ and $\kappa$ is the ratio of the asymptotic time complexities of HCDE to DE.

From equation (26), we can observe that the contribution of the terms related with the complexity of MCS-OPF, dependent on $N S$ and $n p s$, is considerably large for the fulfilment of the inequality conditions. In fact, when using $\mathrm{DE}$, it is commonly accepted to set a size of the population $N P$ not greater than ten times the size of the decision variables, in this case, 10nps [24], making the first two terms of $\kappa$ strongly dependent on the number of scenarios NS. Moreover, given the complexity of the general problem, higher values of NS lead to a better approximation of the objective function via MCS-OPF, i.e., the more likely is to fulfill the condition and the greater can be the reduction of computation time. However, the value of $\varepsilon$ depends on the probability of performing clustering in each generation and at what scale, controlled by $C C C_{t h}$ and $p_{d \% t i l e}$ respectively. In some cases, $\varepsilon$ can be close to $l$ (as we inferred from Figure 11) implying negligible benefits. Table 7 shows the values of the ratio $\kappa$ for each $\left(N P, C C C_{t h}\right.$, $p_{d \%}$ tile) set in HCDE considering the dimensionality of the present case study defined by the values of the parameters $n p s=55, N S=500, N P=50$. The value of $1-\kappa$ can be interpreted as the expected asymptotic relative time reduction achieved by performing HCDE. 


\begin{tabular}{|c|c|c|c|c|c|}
\hline \multirow{2}{*}{$\left(N P, C C C_{t h}, p_{d \% t i l e}\right)$} & $N P$ & $N P \log (N P)$ & $\varepsilon=\underline{\mathrm{E}\left[N P^{G}\right]}$ & $\kappa$ & \\
\hline & $\overline{N S \times n p s}$ & $N S \times n p s^{2}$ & $=\overline{N P}$ & К & $1-\kappa$ \\
\hline$(50,0.6,25)$ & \multirow{9}{*}{$1.818 E-03$} & \multirow{9}{*}{$3.418 E-05$} & 0.817 & 0.819 & 0.181 \\
\hline$(50,0.7,25)$ & & & 0.921 & 0.923 & 0.077 \\
\hline$(50,0.8,25)$ & & & 0.987 & 0.989 & 0.011 \\
\hline$(50,0.6,50)$ & & & 0.510 & 0.512 & 0.488 \\
\hline$(50,0.7,50)$ & & & 0.738 & 0.740 & 0.260 \\
\hline$(50,0.8,50)$ & & & 0.978 & 0.979 & 0.021 \\
\hline$(50,0.6,75)$ & & & 0.259 & 0.261 & 0.739 \\
\hline$(50,0.7,75)$ & & & 0.487 & 0.488 & 0.512 \\
\hline$(50,0.8,75)$ & & & 0.909 & 0.911 & 0.089 \\
\hline
\end{tabular}

441 Figure 12 shows the convergence curves for the DE and HCDE cases selected, for the twenty runs performed for 442 each $\left(N P, C C C_{t h}, p_{d \%}\right)$ setting: no significant differences can be found among the convergence curves except for 443 the expected behavior of converging to lower values of $E G C_{\min }$ for settings which imply a larger population size.
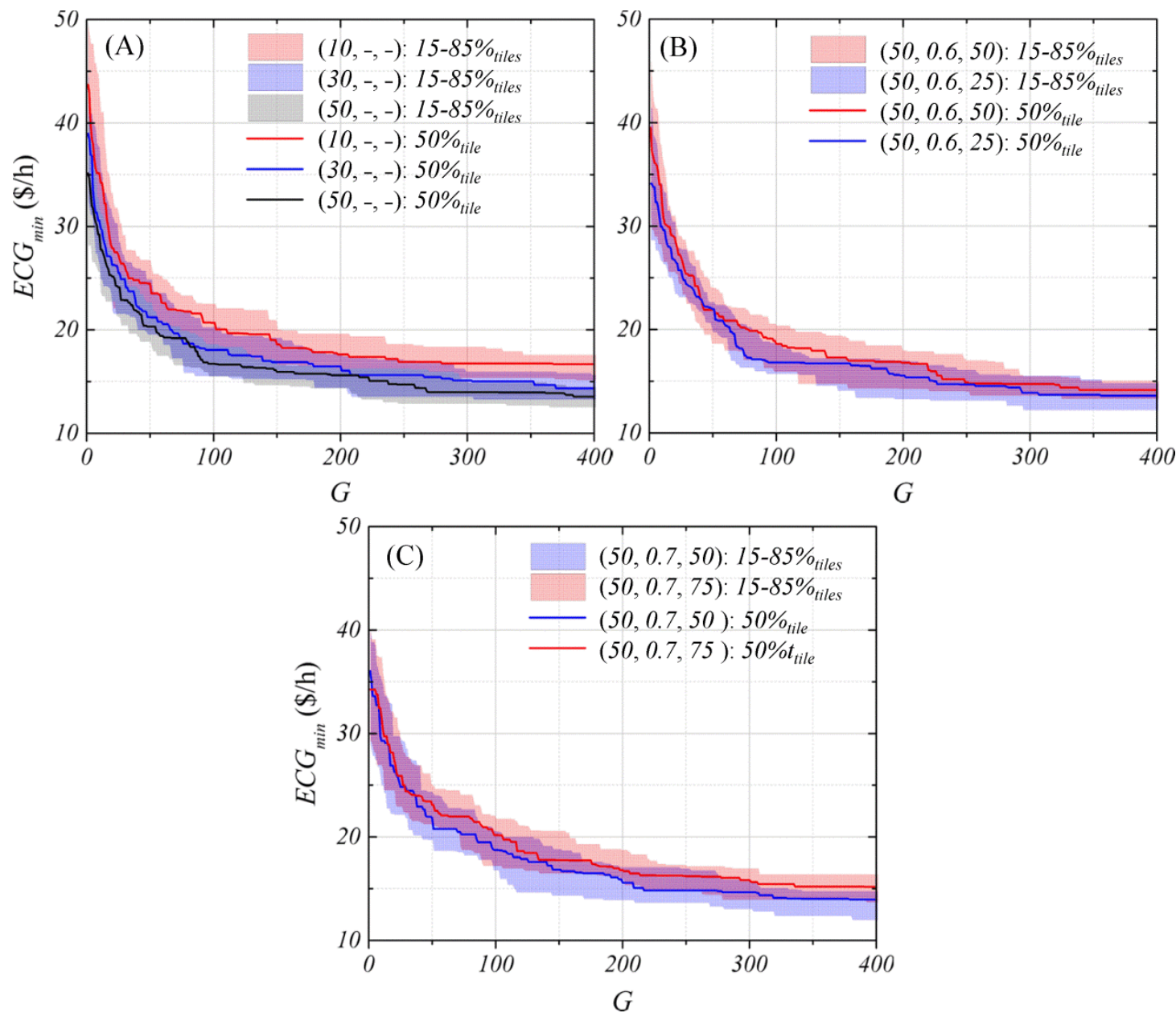

Figure 12. Convergence curves for representative $\left(N P, C C C_{t h}, p_{d \%}\right.$ tile $)$ settings

446 Figure 13 shows the average total DG power allocated in the distribution network and the corresponding investment costs of the DE and HCDE MCS-OPF cases selected, choosing the corresponding optimal DG- 
integrated plans as the decision matrixes $\Xi^{D G}$ for which their $E C G_{\min }$ values are the closest to the median $E C G_{\text {min }}$ value obtained for the twenty runs of each $\left(N P, C C C_{t h}, p_{d \%}\right.$ tile $)$ setting. It can be pointed out that in all the cases, the contribution of $\mathrm{EV}$ is practically negligible if compared with the other technologies. This is due to a combination of two facts: the probability that the EV is in a discharging state is much lower than that of being in the other two possible operating states, charging and disconnected (see Figure 7) and when EV is charging, the effects are opposite to those desired, i.e., it is acting as loads.

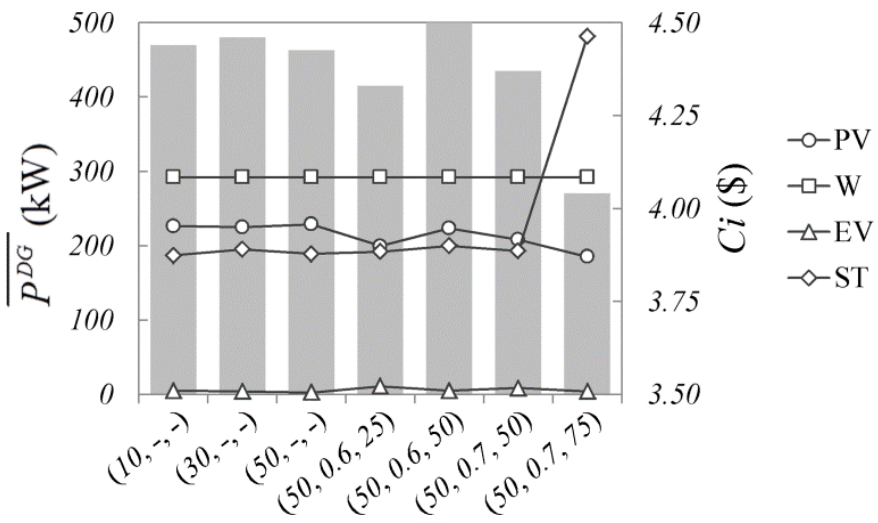

In all generality, both the investment cost $C i$ and the average power installed by DG is comparable in all the cases, except for the setting $(50,0.7,75)$ for which the scale of clustering determined by $p_{d \%}$ tile $=75 \%$, that translates into

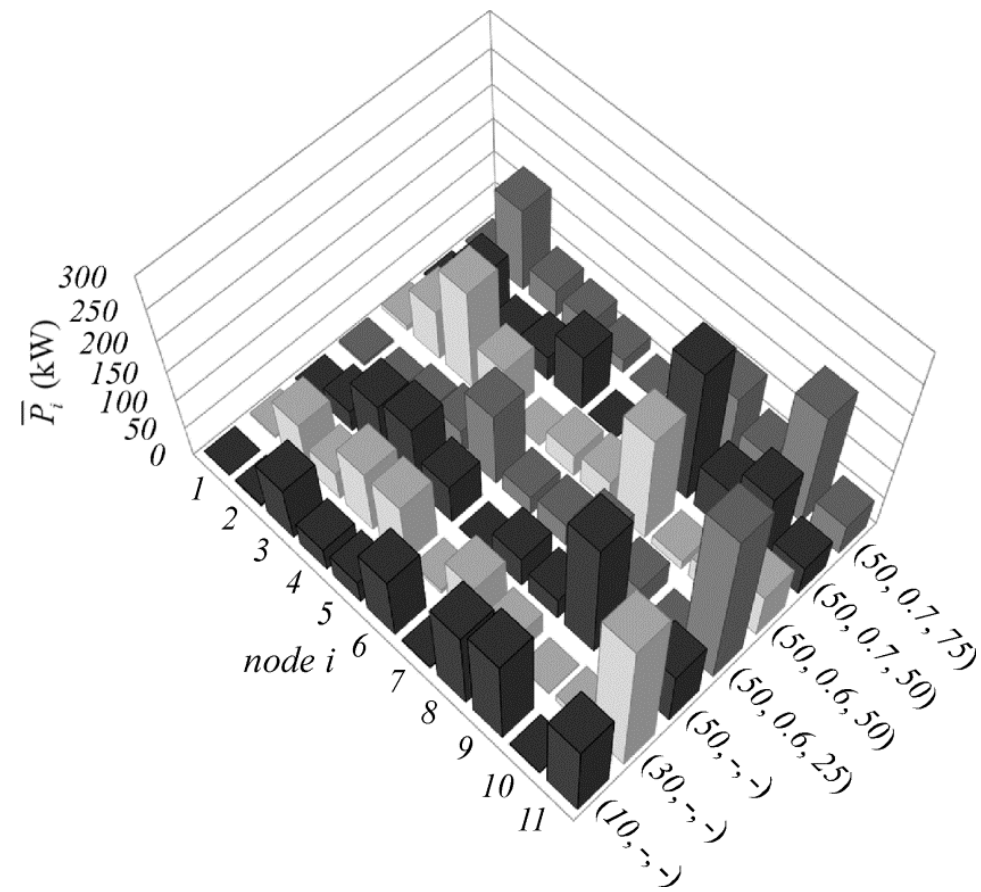


The average total renewable DG power allocated per node is summarized in Figure 14. Even though all the ECG optimal decision matrixes $\Xi^{D G}$ show differences, the tendency is to install localized sources of renewable DG power between two identifiable portions of the distribution network, up and downstream the feeder (2,6) (Figure 5), giving preference to the second portion which presents higher and non-stream homogeneous nodal load profiles.

\section{CONCLUSIONS}

466

467

468

469

470

471

472

473

474

475

476

477

478
In a previous paper, we have presented a simulation and optimization framework for the planning of integration of renewable generation into a distribution network. The optimization is considered with respect the objective of minimizing the expected global cost of the system. The inherent uncertain behavior of renewable energy sources, variability in the main power supply and loads, as well as the possibility of failures of network components are included in a Monte Carlo simulation, which samples realizations of the uncertain operational scenarios for the optimal power flow.

The framework is quite general and complete in the characteristics of the realistic system scenarios considered. However, this is at the expenses of the computational time required for the overall optimization.

In this respect, in the present paper we have addressed the problem of computational efficiency in the resolution of the renewable DG planning optimization problem. We have done so by an original introduction of a controlled clustering strategy, with, the main original contributions being:

- The integration of differential evolution and hierarchical clustering analysis for grouping similar individuals from a given population and selecting representatives to be evaluated for each group, thus reducing the number of objective function evaluations during the optimization.

- The introduction of two control parameters, namely the cophenetic correlation coefficient and a percentile of the set of linkage distances, for allowing controlled adaptation during the search process and decision on whether or not to perform clustering and at which level of the hierarchical structure built.

A case study has been analyzed derived from the IEEE 13 nodes test feeder. The results obtained show the capability of the framework to identify optimal plans of renewable DG integration. The sensitivity analysis over the control parameters of the hierarchical clustering shows that the efficiency is improved with cophenetic correlation thresholds that allow the clustering in almost all generations along the differential evolution, setting the scale of clustering to no more than the fiftieth percentile of the linkage distances in the hierarchical structure proposed. Indeed, this is shown to lead to acceptable reductions in the number of objective function evaluations, with small dispersion and loss of quality in the minimum global cost obtained.

References 
[1] A. Alarcon-Rodriguez, G. Ault, and S. Galloway, "Multi-objective planning of distributed energy resources: A review of the state-of-the-art," Renewable and Sustainable Energy Reviews, vol. 14, pp. 1353 - 1366, 2010.

[2] C. L. T. Borges, "An overview of reliability models and methods for distribution systems with renewable energy distributed generation," Renewable and Sustainable Energy Reviews, vol. 16, pp. 4008-4015, 2012.

[3] V. F. Martins and C. L. T. Borges, "Active Distribution Network Integrated Planning Incorporating Distributed Generation and Load Response Uncertainties," Power Systems, IEEE Transactions on, vol. 26, pp. $2164-2172$, nov. 2011.

[4] H. Ren and W. Gao, "A MILP model for integrated plan and evaluation of distributed energy systems," Applied Energy, vol. 87, pp. 1001 - 1014, 2010.

[5] H. Ren, W. Zhou, K. a. t. Nakagami, W. Gao, and Q. Wu, "Multi-objective optimization for the operation of distributed energy systems considering economic and environmental aspects," Applied Energy, vol. 87, pp. 3642 - 3651, 2010.

[6] C. Chen, S. Duan, T. Cai, B. Liu, and G. Hu, "Optimal Allocation and Economic Analysis of Energy Storage System in Microgrids," Power Electronics, IEEE Transactions on, vol. 26, pp. 2762 -2773, oct. 2011.

[7] S. Haffner, L. F. A. Pereira, L. A. Pereira, and L. S. Barreto, "Multistage model for distribution expansion planning with distributed generation - Part I: Problem formulation," Ieee Transactions on Power Delivery, vol. 23, pp. 915-923, Apr 2008.

[8] S. Haffner, L. F. A. Pereira, L. A. Pereira, and L. S. Barreto, "Multistage model for distribution expansion planning with distributed generation - Part II: Numerical results," Ieee Transactions on Power Delivery, vol. 23, pp. 924-929, Apr 2008.

[9] M. E. Samper and A. Vargas, "Investment Decisions in Distribution Networks Under Uncertainty With Distributed Generation-Part II: Implementation and Results," Ieee Transactions on Power Systems, vol. 28, pp. 2341-2351, Aug 2013.

[10] T. Niknam, S. I. Taheri, J. Aghaei, S. Tabatabaei, and M. Nayeripour, "A modified honey bee mating optimization algorithm for multiobjective placement of renewable energy resources," Applied Energy, vol. 88, pp. 4817-4830, Dec 2011.

[11] S. Ganguly, N. C. Sahoo, and D. Das, "A novel multi-objective PSO for electrical distribution system planning incorporating distributed generation," Energy Systems, vol. 1, pp. 291-337, 2010.

[12] M. Gomez-Gonzalez, A. LÃ $\tilde{A}^{3}$ pez, and F. Jurado, "Optimization of distributed generation systems using a new discrete PSO and OPF," Electric Power Systems Research, vol. 84, pp. 174 - 180, 2012.

[13] K. Zou, A. P. Agalgaonkar, K. M. Muttaqi, and S. Perera, "Multi-objective optimisation for distribution system planning with renewable energy resources," in Energy Conference and Exhibition (EnergyCon), 2010 IEEE International, ed, 2010, pp. 670 -675.

[14] H. A. Hejazi, A. R. Araghi, B. Vahidi, S. H. Hosseinian, M. Abedi, and H. Mohsenian-Rad, "Independent Distributed Generation Planning to Profit Both Utility and DG Investors," Ieee Transactions on Power Systems, vol. 28, pp. 1170-1178, May 2013.

[15] H. A. Hejazi, M. A. Hejazi, G. B. Gharehpetian, and M. Abedi, "Distributed generation site and size allocation through a techno economical multi-objective Differential Evolution Algorithm," in Power and Energy (PECon), 2010 IEEE International Conference on, ed, 2010, pp. 874 -879.

[16] M. F. Shaaban, Y. M. Atwa, and E. F. El-Saadany, "DG Allocation for Benefit Maximization in Distribution Networks," Ieee Transactions on Power Systems, vol. 28, pp. 639-649, May 2013.

[17] M. Raoofat, "Simultaneous allocation of DGs and remote controllable switches in distribution networks considering multilevel load model," International Journal of Electrical Power and Energy Systems, vol. 33, pp. 1429 - 1436, 2011. 
[18] Z. H. Cai, W. Y. Gong, C. X. Ling, and H. Zhang, "A clustering-based differential evolution for global optimization," Applied Soft Computing, vol. 11, pp. 1363-1379, Jan 2011.

[19] M.-Y. Cheng, D.-H. Tran, and Y.-W. Wu, "Using a fuzzy clustering chaotic-based differential evolution with serial method to solve resource-constrained project scheduling problems," Automation in Construction, vol. 37, pp. 88-97, 1// 2014.

[20] G. Liu, Y. X. Li, X. Nie, and H. Zheng, "A novel clustering-based differential evolution with 2 multiparent crossovers for global optimization," Applied Soft Computing, vol. 12, pp. 663-681, Feb 2012.

[21] R. Mukherjee, G. R. Patra, R. Kundu, and S. Das, "Cluster-based differential evolution with Crowding Archive for niching in dynamic environments," Information Sciences, 2014.

[22] S. Song and X. J. Yu, "Multi-peak function optimization using a hierarchical clustering based genetic algorithm," ISDA 2006: Sixth International Conference on Intelligent Systems Design and Applications, Vol 1, pp. 425-428, 2006.

[23] Y. J. Wang, J. S. Zhang, and G. Y. Zhang, "A dynamic clustering based differential evolution algorithm for global optimization," European Journal of Operational Research, vol. 183, pp. 56-73, Nov 162007.

[24] R. Storn and K. Price, "Differential evolution - A simple and efficient heuristic for global optimization over continuous spaces," Journal of Global Optimization, vol. 11, pp. 341-359, Dec 1997.

[25] R. Mena, M. Hennebel, Y. Li, C. Ruiz, and E. Zio, "A Risk-Based Simulation and Multi-Objective Optimization Framework for the Integration of Distributed Renewable Generation and Storage," Renewable and Sustainable Energy Reviews, vol. 37, pp. 778-793, 2014.

[26] B. S. Everitt, S. Landau, M. Leese, and D. Stahl, Cluster Analysis: Wiley, 2011.

[27] IEEE Power and Energy Society. Distribution Test Feeders. Available: http://ewh.ieee.org/soc/pes/dsacom/testfeeders/index.html

[28] H. Falaghi, C. Singh, M.-R. Haghifam, and M. Ramezani, "DG integrated multistage distribution system expansion planning," International Journal of Electrical Power and Energy Systems, vol. 33, pp. 1489 1497, 2011.

[29] Y. M. Atwa, E. F. El-Saadany, M. M. A. Salama, and R. Seethapathy, "Optimal Renewable Resources Mix for Distribution System Energy Loss Minimization," Power Systems, IEEE Transactions on, vol. 25, pp. $360-370$, feb. 2010.

[30] Y. Li and E. Zio, "Uncertainty analysis of the adequacy assessment model of a distributed generation system," Renewable Energy, vol. 41, pp. 235 - 244, 2012.

[31] D. V. Hertem, "Usefulness of DC power flow for active power flow analysis with flow controlling devices," AC and DC Power Transmission, IEEE International Conference on, 2006.

[32] K. Purchala and L. Meeus, "Usefulness of DC power flow for active power flow analysis," Power Engineering and Optimization, 2005.

[33] W. H. Kersting, "Radial distribution test feeders," IEEE Transactions on Power Systems, vol. 6, pp. $975-$ 985, 1991.

[34] L. F. Wang and C. Singh, "Multicriteria Design of Hybrid Power Generation Systems Based on a Modified Particle Swarm Optimization Algorithm," Ieee Transactions on Energy Conversion, vol. 24, pp. 163-172, Mar 2009.

[35] Y.-F. Li and E. Zio, "A multi-state model for the reliability assessment of a distributed generation system via universal generating function," Reliability Engineering \& System Safety, vol. 106, pp. 28-36, 2012.

[36] F. Pilo, G. Celli, S. Mocci, and G. G. Soma, "Active distribution network evolution in different regulatory environments," in Power Generation, Transmission, Distribution and Energy Conversion (MedPower 2010), 7th Mediterranean Conference and Exhibition on, ed, 2010, pp. 1 -8. 
582

583

584

585

586

587
[37] R. Webster, "Can the electricity distribution network cope with an influx of electric vehicles?," Journal of Power Sources, pp. 217-225, 1999.

[38] B. Korte and J. Vygen, Combinatorial Optimization: Theory and Algorithms: Springer Publishing Company, Incorporated, 2007. 